\title{
Unitary subsector of generalized minimal models
}

\author{
Connor Behan \\ C. N. Yang Institute for Theoretical Physics, Stony Brook University, Stony Brook, New York 11794, USA
}

(Received 9 January 2018; published 18 May 2018)

\begin{abstract}
We revisit the line of nonunitary theories that interpolate between the Virasoro minimal models. Numerical bootstrap applications have brought about interest in the four-point function involving the scalar primary of lowest dimension. Using recent progress in harmonic analysis on the conformal group, we prove the conjecture that global conformal blocks in this correlator appear with positive coefficients. We also compute many such coefficients in the simplest mixed correlator system. Finally, we comment on the status of using global conformal blocks to isolate the truly unitary points on this line.
\end{abstract}

DOI: 10.1103/PhysRevD.97.094020

\section{INTRODUCTION}

Conformal field theories (CFTs) in two dimensions enjoy invariance under two copies of the Virasoro algebra—an algebra defined by

$\left[L_{m}, L_{n}\right]=(m-n) L_{m+n}+\frac{c}{12} m(m-1)(m+1) \delta_{m+n, 0}$,

where $c$ is the central charge. The power of this infinitedimensional symmetry was perhaps most famously demonstrated in [1] with the discovery of the minimal models. In addition to providing an exact solution, representation theory of the Virasoro algebra enabled the authors of Refs. [2-5] to show that these models are the only unitary CFTs in two dimensions with $c<1$. However, it has become known more recently that one can see hints of the special role played by minimal models without exploiting Virasoro symmetry at all [6-13]. The method in question is the numerical bootstrap, which uses only the global conformal transformations-two copies of $\mathfrak{g l}(2)$ in this case. Exclusion plots, based on crossing symmetry and unitarity, are shown in Fig. 1 where a straight line containing the minimal models is clearly visible. ${ }^{1}$ A kink is present at the Ising point $\left(\Delta_{\sigma}, \Delta_{\epsilon}\right)=\left(\frac{1}{8}, 1\right)$ but, unlike in the three-dimensional case [14-16], this kink does not sharpen into an island when three correlators are used to restrict the number of relevant operators. Following [17], it

\footnotetext{
${ }^{1}$ The algorithm used to generate Fig. 1 will become important in Sec. IV. These details are summarized in Appendix B.

Published by the American Physical Society under the terms of the Creative Commons Attribution 4.0 International license. Further distribution of this work must maintain attribution to the author(s) and the published article's title, journal citation, and DOI. Funded by SCOAP ${ }^{3}$.
}

is worthwhile to see which features of the $\frac{1}{8} \leq \Delta_{\sigma} \leq \frac{1}{2}$ solution can be predicted analytically. The goal of this work is to put the one-correlator upper bound on a more rigorous footing and to explain why the three-correlator upper bound is unchanged.

In order to have a unitary two-dimensional (2D) CFT with $c<1$, it is necessary that all primary operators have conformal weights equal to Kac's formula $h_{r, s}(c)$ for some $(r, s)$. The Kac table of degenerate weights is given by

$$
\begin{aligned}
c & =1-\frac{6}{m(m+1)} \quad m>2, \\
h_{r, s} & =\frac{[(m+1) r-m s]^{2}-1}{4 m(m+1)} \quad r, s \in \mathbb{Z}_{>0} .
\end{aligned}
$$

Each of these Verma modules has a null state at level $r s$. In the operator product expansion (OPE) of primary operators $\phi_{r, s}$ and $\phi_{r^{\prime}, s^{\prime}}$, the new conformal families that appear are captured in the fusion rule

$$
\phi_{r, s} \times \phi_{r^{\prime}, s^{\prime}}=\sum_{k=0}^{\left\lfloor\frac{r+r^{\prime}-\left|r-r^{\prime}\right|-2}{2}\right\rfloor} \sum_{l=0}^{\left\lfloor\frac{s+s^{\prime}-\left|s-s^{\prime}\right|-2}{2}\right\rfloor} \phi_{\left|r-r^{\prime}\right|+2 k+1,\left|s-s^{\prime}\right|+2 l+1} .
$$

For generic values of $m$, this leads to an infinite discrete spectrum. All OPEs are finite, but as we raise the values of $r$ and $s$, these sums become arbitrarily long. A special situation occurs when $m$ is an integer. This precisely describes a central charge for which $h_{r, s}(c)=h_{m-r, m+1-s}(c)$. The Kac table for these doubly degenerate weights can be shown to truncate, allowing us to consider only $0<r<m$ and $0<s<m+1$. This leads to a finite number of primary operators and, as it turns out, a unitary theory. This theory, called a (unitary) minimal model, is often denoted $\mathcal{M}(m+1, m)$. Since Fig. 1 only shows a kink for 


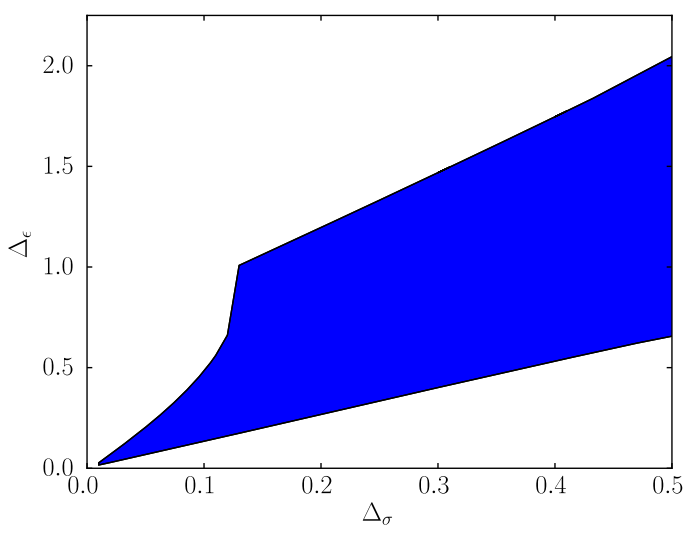

(a) One correlator

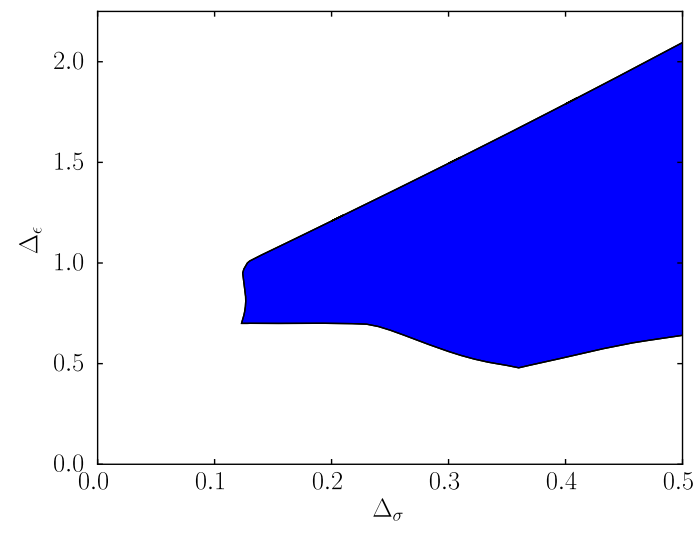

(b) Three correlators

FIG. 1. Allowed regions for the dimensions of $\sigma$ and $\epsilon$ - the $\mathbb{Z}_{2}$-odd scalar of smallest dimension and the $\mathbb{Z}_{2}$-even scalar of smallest dimension, respectively. In both cases, all OPEs are restricted to contain only one relevant scalar. (a) The constraints of crossing symmetry and unitarity on the four-point function $\langle\sigma \sigma \sigma \sigma\rangle$. (b) The constraints of crossing symmetry and unitarity on $\langle\sigma \sigma \sigma \sigma\rangle,\langle\sigma \sigma \epsilon \epsilon\rangle$, and $\langle\epsilon \epsilon \epsilon \epsilon\rangle$.

$m=3$, it is evident that noninteger values of $m$ are still important for the bootstrap. Analytically continuing the unitary minimal models in this way is not new. In $[18,19]$, the generic $\mathcal{M}(m+1, m)$ was found to be a solvable consistent theory and referred to as a generalized minimal model. ${ }^{2}$ It obeys the 2D CFT axioms of associativity and Virasoro symmetry but not unitary [20]. The upper bound from the bootstrap

$$
\Delta_{\epsilon}=\frac{1}{3}\left(8 \Delta_{\sigma}+2\right)
$$

is realized by the generalized minimal model four-point function $\langle\sigma \sigma \sigma \sigma\rangle$ if we identify $\sigma \equiv \phi_{1,2}$ and $\epsilon \equiv \phi_{1,3} \cdot{ }^{3}$ The authors of [17] observed squared OPE coefficients of quasiprimaries in this correlator that were all positive. If this conjecture is correct, we must conclude that in an arbitrary $\mathcal{M}(m+1, m)$ theory, the nonunitarity is mild enough that it cannot be diagnosed from the correlator of four $\sigma$ operators. What this means is that for a nondegenerate $\mathcal{O}$ appearing in the $\sigma \times \sigma$ OPE, the state $\mathcal{O}\left(x_{1}\right) \sigma\left(x_{2}\right) \sigma\left(x_{3}\right)|0\rangle$ on the cylinder will have a positive norm. Similarly, within a degenerate subspace, all such norms will sum to a positive number. We use the terminology that $\langle\sigma \sigma \sigma \sigma\rangle$ comprises a unitary subsector of the theory since other OPEs, e.g. $\sigma \times \epsilon$, are needed to construct negative norms. As we will show, the conjecture can indeed be proven with the help of a new formalism in [21,22] for deriving $\mathfrak{g l}(2)$ block expansions. This proof, along with a systematic look at the other two correlators, forms the main

\footnotetext{
${ }^{2}$ This should not be confused with the nonunitary minimal model, which describes a nonunitary $c<1$ theory with finitely many primaries. This discrete set is denoted $\mathcal{M}(p, q)$ with $p$ and $q$ relatively prime.

${ }^{3}$ This notation differs from the statistical physics literature in which it is natural to regard $\phi_{2,2}$ as the spin field.
}

result of this work. ${ }^{4}$ The summary of results in Table I shows that for $3<m<4,\langle\sigma \sigma \sigma \sigma\rangle$ is the only unitary subsector we have found in the sense described above. However, it appears that the generalized minimal models with $m>4$ have larger unitary subsectors that include the other two four-point functions. The last line shows a surprising tension with Fig. 1. Despite the fact that $\langle\epsilon \epsilon \epsilon \epsilon\rangle$ displays significant unitarity violation for $m<4$, the line (1.4) in the three-correlator exclusion plot is uninterrupted. The numerics are telling us that there is a partial solution to crossing, other than $\mathcal{M}(m+1, m)$, which fills in this region. Using the properties of minimal models, we will show that the existence of this solution can be concluded from a simpler numerical setup. It would be nice to eventually find a fully analytic construction.

Before deriving the results in Table I, it is instructive to consider the lower bound

$$
\Delta_{\epsilon}=\frac{4}{3} \Delta_{\sigma}
$$

which appears on the left side of Fig. 1. This gives us a more straightforward opportunity to use the techniques in $[21,22]$. The explicit solution for $\langle\sigma \sigma \sigma \sigma\rangle$ along this line was found in [23], which focused on its special role in the nonunitary (severe truncation) bootstrap of $[24-26] .{ }^{5}$ This

${ }^{4}$ Looking ahead, the decompositions (3.11) and (3.10) are essential for the positivity proof. We have learned that they were previously obtained, through a slightly different method, in unpublished work by Mikhail Isachenkov and Volker Schomerus.

${ }^{5}$ The fact that it also appears in the unitary bootstrap has not received much attention. In [27], it was mentioned that the lower bound at $\Delta_{\sigma}=\frac{1}{8}$ was somewhat close to $\Delta_{\epsilon}=\frac{1}{6}$. Interestingly, the region below the bound coincides with the region where standard OPE maximization techniques cannot constrain the central charge [28]. 
TABLE I. The status of three-correlator $\mathfrak{g l}(2)$ block coefficients in the generalized minimal models. Statements about $\langle\sigma \sigma \sigma \sigma\rangle$ apply rigorously to the full set of coefficients. For the other two correlators, we have manually decomposed them up to order 15 .

\begin{tabular}{lll}
\hline \hline Correlator & \multicolumn{1}{c}{$3<m<4$} & $4<m<\infty$ \\
\hline$\langle\sigma \sigma \sigma \sigma\rangle$ & All coefficients $\geq 0$ & All coefficients $\geq 0$ \\
$\langle\sigma \sigma \epsilon \epsilon\rangle$ & One checked coefficient $<0$ & All checked coefficients $\geq 0$ \\
$\langle\epsilon \epsilon \epsilon \epsilon\rangle$ & Infinitely many coefficients $<0$ & All checked coefficients $\geq 0$ \\
\hline \hline
\end{tabular}

solution exhibits Virasoro symmetry with a central charge given by

$$
c=1+16 \Delta_{\sigma}
$$

but no Virasoro identity block. To find an analytic explanation for why this four-point function appears in the unitary bootstrap, one must be able to show that this vacuum decoupling is the only sign of nonunitarity that appears at the level of a single correlator. In other words, one must be able to repeat the logic of [17] and find positive squared OPE coefficients for all of the quasiprimaries that do appear. These will be seen as very large coefficients by the numerics because the algorithm used for Fig. 1 fixes $\lambda_{\sigma \sigma I}=1$. This is indeed what we find from the extremal functional method of [29].

This paper is organized as follows. In Sec. II, we study the lower bound (1.5) as a warm-up. In this case, it is particularly easy to invert the OPE and find that all $\mathfrak{s l}(2)$ block coefficients are positive. The methods involved prepare us for our main interest, which is the upper bound (1.4). Turning to this upper bound in Sec. III, we calculate the global block coefficients summarized in Table I. In the case of $\langle\sigma \sigma \sigma \sigma\rangle$, we find closed-form expressions. In showing that they are positive, we prove the conjecture made in the appendix of [17]. For the other two correlators, we expand them to high order recursively and conjecture that the $\mathfrak{g l}(2)$ block coefficients are positive again when $4<m<\infty$. Even though the Virasoro blocks in $\langle\epsilon \epsilon \epsilon \epsilon\rangle$ appear to have positive $\mathfrak{s l}(2)$ expansions everywhere, the unitarity violation for $3<m<4$ arises because of the coefficients multiplying the Virasoro blocks themselves. In Sec. IV, we go back to the bootstrap and discuss what these patterns in the OPE coefficients mean for the results in Fig. 1. In particular, we perform a semianalytic treatment of the problematic correlator. The result is that one does not need to perform a three-correlator bootstrap to predict that (1.4) survives - the search for an upper bound may be reduced to a onecorrelator problem. Before concluding, in Sec. V, we discuss other 2D theories which might be possible to study using more correlators or more assumptions on the spectrum.

\section{THE LOWER LINE: A WARM-UP}

The four-point function along the line (1.5) consists of a single Virasoro block $V\left(h_{i}, h, c ; z\right)$. It was found in [23] via the Coulomb gas formalism which writes the central charge as $c=1-24 \alpha_{0}^{2}$ and places a background charge of $2 \alpha_{0}$ at infinity. This allows a number of four-point functions to be realized as correlators of vertex operators with additional insertions of screening charges. The simplest of these is a correlator of four scalars that all have charge $\frac{\alpha_{0}}{2}$. Since the neutrality condition for this is satisfied without any screening charges, one finds the manifestly crossing symmetric

$$
\begin{aligned}
& \langle\sigma(0) \sigma(z, \bar{z}) \sigma(1) \sigma(\infty)\rangle \\
& =V\left(-\frac{3}{4} \alpha_{0}^{2},-\alpha_{0}^{2}, 1-24 \alpha_{0}^{2} ; z\right) V\left(-\frac{3}{4} \alpha_{0}^{2},-\alpha_{0}^{2}, 1-24 \alpha_{0}^{2} ; \bar{z}\right) \\
& =|z(1-z)|^{\alpha_{0}^{2}},
\end{aligned}
$$

where we have used $h=\alpha\left(\alpha-2 \alpha_{0}\right)$. Expressing (2.1) in terms of $\Delta_{\sigma}$,

$$
\begin{aligned}
\langle\sigma(0) \sigma(z, \bar{z}) \sigma(1) \sigma(\infty)\rangle & =\frac{g(z) g(\bar{z})}{|z|^{2 \Delta_{\sigma}}}, \\
g(z) & =z^{\frac{2}{3} \Delta_{\sigma}}(1-z)^{-\frac{1}{3} \Delta_{\sigma}} .
\end{aligned}
$$

Our task now is to expand $g(z)$ into $\mathfrak{g l}(2)$ blocks:

$$
\begin{aligned}
g(z) & =\sum_{n=0}^{\infty} c_{n} K_{\frac{2}{3} \Delta_{\sigma}+n}(z), \\
K_{h}(z) & \equiv z_{2}^{h} F_{1}(h, h ; 2 h ; z) .
\end{aligned}
$$

This is guaranteed to be an expansion in even integers due to the Bose symmetry of the $\sigma \times \sigma$ OPE. To proceed by the brute-force approach, we expand the hypergeometric function and switch the order of two sums.

$$
\begin{aligned}
g(z) & =\sum_{n=0}^{\infty} \sum_{m=0}^{\infty} c_{n} \frac{\left(\frac{2}{3} \Delta_{\sigma}+n\right)_{m}^{2}}{\left(\frac{4}{3} \Delta_{\sigma}+2 n\right)_{m}} \frac{z^{\frac{2}{3} \Delta_{\sigma}+n+m}}{m !} \\
& =\sum_{k=0}^{\infty} \sum_{n=0}^{k} c_{n} \frac{\left(\frac{2}{3} \Delta_{\sigma}+n\right)_{k-n}^{2}}{\left(\frac{4}{3} \Delta_{\sigma}+2 n\right)_{k-n}} \frac{z^{\frac{2}{3} \Delta_{\sigma}+k}}{(k-n) !} .
\end{aligned}
$$

We may now compare the inner finite sums to the Taylor coefficients of (2.2), given by $b_{k}=\frac{\left(\frac{1}{3} \Delta_{\sigma}\right)_{k}}{k !}$. Since the lower triangular system for $c_{n}$ yields to back-substitution, 


$$
c_{2 k}=b_{2 k}-\sum_{n=0}^{k-1} c_{2 n} \frac{\left(\frac{2}{3} \Delta_{\sigma}+2 n\right)_{2(k-n)}^{2}}{\left(\frac{4}{3} \Delta_{\sigma}+4 n\right)_{2(k-n)}} \frac{1}{(2 k-2 n) !} .
$$

Rather than using this recursive procedure, we will now review a method for computing the $c_{n}$ directly. The blocks, defined in (2.3), are eigenfunctions of the conformal Casimir

$$
\begin{aligned}
D K_{h}(z) & =h(h-1) K_{h}(z), \\
D & =z^{2}(1-z) \frac{\partial^{2}}{\partial z^{2}}-z^{2} \frac{\partial}{\partial z} .
\end{aligned}
$$

It is well known that $D$ is self-adjoint on $[0,1]$ with respect to the measure $z^{-2}$. The authors of [21] used this fact to develop the Sturm-Liouville theory of this operator and construct the orthogonal eigenfunctions

$$
\Psi_{h}(z)=\frac{\Gamma(1-2 h)}{\Gamma(1-h)^{2}} K_{h}(z)+(h \leftrightarrow 1-h) .
$$

It is convenient to set $h=\frac{1}{2}+\alpha$ in which case (2.7) becomes a function $\Psi_{\alpha}(z)$ which is even in $\alpha$. In order for it to have a finite norm, $\alpha$ cannot be real. We must go to imaginary dimension space and take $\alpha \in i \mathbb{R}$. ${ }^{6}$ The result is that with any four-point function $f(z)$, we may associate a density $\hat{f}(\alpha)=\hat{f}(-\alpha)$ via the invertible transform

$$
\begin{aligned}
f(z) & =\frac{1}{2 \pi i} \int_{-i \infty}^{i \infty} \hat{f}(\alpha) \Psi_{\alpha}(z) \frac{d \alpha}{N(\alpha)}, \\
N(\alpha) & \equiv \frac{\Gamma(\alpha) \Gamma(-\alpha)}{2 \pi \Gamma\left(\frac{1}{2}+\alpha\right) \Gamma\left(\frac{1}{2}-\alpha\right)} .
\end{aligned}
$$

It is now clear that OPE coefficients may be read off from the residues of $\hat{f}(\alpha)$ whenever its poles are on the real axis. A formula that $[21,22]$ derived using this method is

$$
\begin{aligned}
z^{p}(1-z)^{-q}= & \sum_{n=0}^{\infty} \frac{(p)_{n}^{2}}{(2 p+n-1)_{n} n !}{ }_{3} F_{2} \\
& \times\left(\begin{array}{c}
-n, 2 p+n-1, p-q \\
p, p
\end{array}\right) K_{p+n}(z) .
\end{aligned}
$$

We will use this in the current section and the next one.

Specializing (2.9) to the four-point function (2.2), we immediately find

$c_{n}=\frac{\left(\frac{2}{3} \Delta_{\sigma}\right)_{n}^{2}}{\left(\frac{4}{3} \Delta_{\sigma}+n-1\right)_{n} n !}{ }_{3} F_{2}\left(\begin{array}{c}-n, \frac{4}{3} \Delta_{\sigma}+n-1, \frac{1}{3} \Delta_{\sigma} \\ \frac{2}{3} \Delta_{\sigma}, \frac{2}{3} \Delta_{\sigma}\end{array}\right)$.

There are two ways to assess the positivity of (2.10). The first is to recall the definition of a continuous Hahn polynomial [30],

$$
\begin{aligned}
& \tilde{P}_{n}(a, b, c, d ; x) \\
& ={ }_{3} F_{2}\left(\begin{array}{c}
-n, n+a+b+c+d-1, a+x \\
a+c, a+d
\end{array} ; 1\right) .
\end{aligned}
$$

Clearly,

$$
c_{n}=\frac{\left(\frac{2}{3} \Delta_{\sigma}\right)_{n}^{2}}{\left(\frac{4}{3} \Delta_{\sigma}+n-1\right)_{n} n !} \tilde{P}_{n}\left(\frac{2}{3} \Delta_{\sigma}, \frac{2}{3} \Delta_{\sigma}, 0,0 ;-\frac{1}{3} \Delta_{\sigma}\right)
$$

is a valid rewriting of (2.10). ${ }^{7}$ Suppressing their parameters, the polynomials $\tilde{P}_{n}(a, b, c, d ; x)$ satisfy the following recurrence relation:

$$
\begin{aligned}
(x+a) \tilde{P}_{n}(x) & =A_{n} \tilde{P}_{n+1}(x)-\left(A_{n}+B_{n}\right) \tilde{P}_{n}(x)+B_{n} \tilde{P}_{n-1}(x), \\
A_{n} & \equiv-\frac{(n+a+b+c+d-1)(n+a+c)(n+a+d)}{(2 n+a+b+c+d-1)(2 n+a+b+c+d)}, \\
B_{n} & \equiv \frac{n(n+b+c-1)(n+b+d-1)}{(2 n+a+b+c+d-2)(2 n+a+b+c+d-1)} .
\end{aligned}
$$

For our parameters, we may easily check that $A_{n}+B_{n}+a+x=0$. It then follows by induction that all $c_{2 k+1}$ vanish. Once we know this, Eq. (2.13) is effectively a two-term recursion. Seeing a positive constant of proportionality in

$$
\begin{aligned}
\tilde{P}_{2 k}\left(-\frac{1}{3} \Delta_{\sigma}\right) & =-\frac{B_{2 k-1}}{A_{2 k-1}} \tilde{P}_{2 k-2}\left(-\frac{1}{3} \Delta_{\sigma}\right) \\
& =\frac{3(2 k-1)\left(\Delta_{\sigma}+3 k-3\right)}{\left(2 \Delta_{\sigma}+3 k-3\right)\left(2 \Delta_{\sigma}+6 k-3\right)} \tilde{P}_{2 k-2}\left(-\frac{1}{3} \Delta_{\sigma}\right),
\end{aligned}
$$

\footnotetext{
${ }^{6}$ There is another name for this space as evidenced by the title of [21].

${ }^{7}$ In the notation of [31], we would write $c_{n}=\frac{2^{-n}}{n !} Q_{n, 0}^{\frac{4}{3} \Delta_{\sigma}+n}\left(-\frac{1}{3} \Delta_{\sigma}\right)$.
} 
we conclude that the sequence $c_{2 k}$ decays to zero monotonically from above. It is therefore imperative that the bootstrap single out a region that includes (1.5). To derive this without referring to continuous Hahn polynomials, one may instead express $c_{n}$ in terms of gamma functions. This is possible because Watson's theorem [32],

$$
{ }_{3} F_{2}\left(\begin{array}{c}
a, b, c \\
\frac{a+b+1}{2}, 2 c
\end{array} ; 1\right)=\frac{\Gamma\left(\frac{1}{2}\right) \Gamma\left(\frac{1+a+b}{2}\right) \Gamma\left(\frac{1}{2}+c\right) \Gamma\left(\frac{1-a-b}{2}+c\right)}{\Gamma\left(\frac{1+a}{2}\right) \Gamma\left(\frac{1+b}{2}\right) \Gamma\left(\frac{1-a}{2}+c\right) \Gamma\left(\frac{1-b}{2}+c\right)},
$$

applies whenever $p=2 q$ in (2.9). Although $\Re(a+b-2 c)<1$ is usually needed for convergence, we may drop this requirement for a hypergeometric function that terminates.

This analysis does not explain why (1.5) saturates the lower bound in the one-correlator result of Fig. 1. However, it is encouraging that bounds of this form in one dimension have been proven in [33]. The Coulomb gas formalism does not yield an obvious way to solve for the correlators $\langle\sigma \sigma \epsilon \epsilon\rangle,\langle\epsilon \epsilon \epsilon \epsilon\rangle$ or even to verify that they exist. Because the three-correlator plot in Fig. 1 excludes this line, any theory to which $\langle\sigma \sigma \sigma \sigma\rangle$ could extend would have to be highly nonunitary.

In this section, we have seen two methods for proving that global block coefficients in (2.2) are positive. One uses Watson's theorem and the other uses a recurrence relation for orthogonal polynomials. We will need both of these methods when we prove positivity in the generalized minimal models. Before moving on, there is an interesting way to check our results in a spacetime with Minkowski signature. Even though (2.2) is not strictly a correlation function in a unitary theory, it is still bounded in the Regge limit. Its $\mathfrak{g l}(2)$ block expansion should therefore be calculable with the conformal Froissart-Gribov formula [34] which in our case reads

$$
\begin{aligned}
c(\Delta, \ell)= & \kappa_{\Delta+\ell} \int_{0}^{1} \int_{0}^{1} K_{\frac{\Delta+\ell}{2}}(z) K_{\frac{\ell-\Delta+2}{2}}(\bar{z}) \mathrm{dDisc}\left[|z|^{2 \Delta_{\sigma}}\right. \\
& <\sigma(0) \sigma(z, \bar{z}) \sigma(1) \sigma(\infty)>] \frac{\mathrm{d} z}{z^{2}} \frac{\mathrm{d} \bar{z}}{\bar{z}^{2}} \\
\kappa_{\beta} \equiv & \frac{\Gamma\left(\frac{\beta}{2}\right)^{4}}{2 \pi^{2} \Gamma(\beta-1) \Gamma(\beta)} .
\end{aligned}
$$

To define the double discontinuity, we must treat $z, \bar{z}$ as independent variables and rotate around the $\bar{z}=1$ branch point. Since this can be done in two ways, we subtract the average from our four-point function to find

$$
\mathrm{dDisc}[g(z) g(\bar{z})]=2 \sin ^{2}\left(\frac{\pi \Delta_{\sigma}}{3}\right)(z \bar{z})^{\frac{2}{3} \Delta_{\sigma}}[(1-z)(1-\bar{z})]^{-\frac{1}{3} \Delta_{\sigma}} .
$$

Performing the factored integrals yields a spectral density given by

$$
\begin{aligned}
& \frac{c(\Delta, \ell) \Gamma\left(1-\frac{1}{3} \Delta_{\sigma}\right)^{-2}}{2 \sin ^{2}\left(\frac{\pi \Delta_{\sigma}}{3}\right) \kappa_{\Delta+\ell}}=\frac{\Gamma\left(\frac{2}{3} \Delta_{\sigma}+\frac{\ell-\Delta}{2}\right)}{\Gamma\left(\frac{1}{3} \Delta_{\sigma}+\frac{\ell-\Delta+2}{2}\right)}{ }_{3} F_{2}\left(\begin{array}{l}
\frac{\ell-\Delta+2}{2}, \frac{\ell-\Delta+2}{2}, \frac{2}{3} \Delta_{\sigma}+\frac{\ell-\Delta}{2} \\
\ell-\Delta+2, \frac{1}{3} \Delta_{\sigma}+\frac{\ell-\Delta+2}{2} ; 1
\end{array}\right) \\
& \times \frac{\Gamma\left(\frac{2}{3} \Delta_{\sigma}+\frac{\Delta+\ell-2}{2}\right)}{\Gamma\left(\frac{1}{3} \Delta_{\sigma}+\frac{\Delta+\ell}{2}\right)}{ }_{3} F_{2}\left(\begin{array}{c}
\frac{\Delta+\ell}{2}, \frac{\Delta+\ell}{2}, \frac{2}{3} \Delta_{\sigma}+\frac{\Delta+\ell-2}{2} \\
\Delta+\ell, \frac{1}{3} \Delta_{\sigma}+\frac{\Delta+\ell}{2}
\end{array} ; 1\right)
\end{aligned}
$$

with poles at $\Delta-\ell=\frac{4}{3} \Delta_{\sigma}+2 n$. As none of these are integers, the correct prescription for finding OPE coefficients is to simply take the residue [34],

$$
\begin{aligned}
-\operatorname{Res}(c(\Delta, \ell), n)= & \frac{(-1)^{n}}{n !} \frac{8 \sin ^{2}\left(\frac{\pi \Delta_{\sigma}}{3}\right) \kappa_{\frac{4}{3} \Delta_{\sigma}+2 \ell+2 n} \Gamma\left(1-\frac{1}{3} \Delta_{\sigma}\right)^{2} \Gamma\left(\frac{4}{3} \Delta_{\sigma}+n+\ell-1\right)}{\Gamma\left(\Delta_{\sigma}+\ell+n\right) \Gamma\left(1-\frac{1}{3} \Delta_{\sigma}-n\right)} \\
& \times{ }_{3} F_{2}\left(\begin{array}{c}
\frac{4}{3} \Delta_{\sigma}+\ell+n-1, \frac{2}{3} \Delta_{\sigma}+\ell+n, \frac{2}{3} \Delta_{\sigma}+\ell+n \\
\Delta_{\sigma}+\ell+n, \frac{4}{3} \Delta_{\sigma}+2 \ell+2 n
\end{array} ; 1\right) \\
& \times{ }_{3} F_{2}\left(\begin{array}{c}
-n, 1-n-\frac{2}{3} \Delta_{\sigma}, 1-n-\frac{2}{3} \Delta_{\sigma} ; 1 \\
1-n-\frac{1}{3} \Delta_{\sigma}, 2-2 n-\frac{4}{3} \Delta_{\sigma}
\end{array}\right) .
\end{aligned}
$$

Although it is not obvious, we have checked that (2.19) is equal to $c_{n} c_{n+\ell}$ by using Watson's theorem twice.

\section{THE UPPER LINE: ONE AND THREE CORRELATORS}

Each state $\left|h_{r, s}\right\rangle$ dual to a degenerate operator in a generalized minimal model has a null descendant $\left|\chi_{r, s}\right\rangle$ at level rs called a singular vector. Four-point functions may be calculated once the necessary singular vectors are known. We simply convert $\chi_{r, s}$ to a differential operator with the Ward identities 
TABLE II. Operators that can appear in $\langle\sigma \sigma \sigma \sigma\rangle,\langle\sigma \sigma \epsilon \epsilon\rangle,\langle\epsilon \epsilon \epsilon \epsilon\rangle$, and their holomorphic weights. The fusion rules would shorten e.g. in the Ising model $m=3$ and tricritical Ising model $m=4$, but we are interested in $\mathcal{M}(m+1, m)$ for real $m$.

\begin{tabular}{ll}
\hline \hline Fusion rules & \multicolumn{1}{c}{ Weights } \\
\hline$\phi_{1,2} \times \phi_{1,2}=\phi_{1,1}+\phi_{1,3}$ & $h_{1,1}=0$ \\
$\phi_{1,2} \times \phi_{1,3}=\phi_{1,2}+\phi_{1,4}$ & $h_{1,2}=\frac{\Delta_{\sigma}}{2}$ \\
$\phi_{1,3} \times \phi_{1,3}=\phi_{1,1}+\phi_{1,3}+\phi_{1,5}$ & $h_{1,3}=\frac{4 \Delta_{\sigma}+1}{3} \equiv \frac{\Delta_{\epsilon}}{2}$ \\
& $h_{1,4}=\frac{5 \Delta_{\sigma}+2}{2}$ \\
& $h_{1,5}=4 \Delta_{\sigma}+2$ \\
\hline \hline
\end{tabular}

$$
\begin{aligned}
L_{-n} & \mapsto \mathcal{L}_{-n}=\sum_{i \neq 1} \frac{h_{i}(n-1)}{\left(z_{i}-z_{1}\right)^{n}}-\frac{\partial_{i}}{\left(z_{i}-z_{1}\right)^{n-1}}, \\
L_{-1} & \mapsto \mathcal{L}_{-1}=\partial_{1},
\end{aligned}
$$

and use the fact that this operator must annihilate any correlation function involving $\phi_{r, s}$ in the first position. The resulting equation, known as a Belavin-PolyakovZamolodchikov (BPZ) differential equation [1], has $r s$ linearly independent solutions representing the exchanged multiplets. One may read off their dimensions by looking at the $O\left(z^{h}\right)$ behavior as $z \rightarrow 0$. While expressions for singular vectors are generally nontrivial, with some appearing only recently [35-37], the ones we need are relatively simple:

$$
\begin{aligned}
\left|\chi_{1, s}\right\rangle= & \sum_{p_{1}+\cdots+p_{k}=s} \frac{(-t)^{s-k}[(s-1) !]^{2}}{\prod_{i=1}^{k-1}\left(p_{1}+\cdots+p_{i}\right)\left(s-p_{1}-\cdots-p_{i}\right)} \\
& \times L_{-p 1} \cdots L_{-p_{k}}\left|h_{1, s}\right\rangle, \\
t \equiv & \frac{m}{m+1} .
\end{aligned}
$$

It is clear that we may confine ourselves to the border of the Kac table when studying correlators of $\sigma \equiv \phi_{1,2}$ and $\epsilon \equiv \phi_{1,3}$. The set of operators $\phi_{1, s}$ which closes under fusion is called the Verlinde subalgebra. We will continue to parametrize the generalized minimal model by $\Delta_{\sigma}$-the horizontal axis of Fig. 1. For convenience, Table II summarizes the OPEs that are important for the simplest mixed correlator system.

\section{A. All global block coefficients in the simplest correlator}

We will now derive new expressions for the squared OPE coefficients in $\langle\sigma \sigma \sigma \sigma\rangle$ along (1.4). Positivity, as predicted by [17], will then follow from methods analogous to those in the last section. Since this correlator solves a secondorder BPZ equation, we may write it as

$$
\begin{aligned}
& \left\langle\sigma\left(z_{1}, \bar{z}_{1}\right) \sigma\left(z_{2}, \bar{z}_{2}\right) \sigma\left(z_{3}, \bar{z}_{3}\right) \sigma\left(z_{4}, \bar{z}_{4}\right)\right\rangle=\frac{G(z, \bar{z})}{\left|z_{12} z_{34}\right|^{2 \Delta_{\sigma}}}, \\
& G(z, \bar{z})=G_{(1,1)}^{\sigma \sigma \sigma \sigma}(z) G_{(1,1)}^{\sigma \sigma \sigma \sigma}(\bar{z})+C_{(1,2)(1,2)}^{(1,3)} G_{(1,3)}^{\sigma \sigma \sigma \sigma}(z) G_{(1,3)}^{\sigma \sigma \sigma \sigma}(\bar{z}),
\end{aligned}
$$

where the functions of $z$ are Virasoro blocks. ${ }^{8}$ The specific operator, read off from (3.2), is

$$
\frac{3}{2\left(\Delta_{\sigma}+1\right)} \mathcal{L}_{-1}^{2}-\mathcal{L}_{-2}
$$

Acting on (3.3) with (3.4), we arrive at a PDE in terms of $\left(z_{1}, z_{2}, z_{3}, z_{4}\right)$. To reduce it to an Ordinary differential equation (ODE), we map these points to $(0, z, 1, \infty)$ by a global conformal transformation:

$$
\begin{aligned}
& \frac{3}{2} z(z-1)^{2} \frac{\partial^{2} G}{\partial z^{2}}+(z-1)\left[\left(2-\Delta_{\sigma}\right) z+2 \Delta_{\sigma}-1\right] \frac{\partial G}{\partial z} \\
& -\frac{1}{2} \Delta_{\sigma}\left(\Delta_{\sigma}+1\right) z G=0 .
\end{aligned}
$$

Well known solutions, which have the expected asymptotic behavior, are

$$
\begin{aligned}
& G_{(1,1)}^{\sigma \sigma \sigma \sigma}(z)=(1-z)^{-\Delta_{\sigma}} F_{2}\left(-2 \Delta_{\sigma}, \frac{1-2 \Delta_{\sigma}}{3} ; \frac{2-4 \Delta_{\sigma}}{3} ; z\right), \\
& G_{(1,3)}^{\sigma \sigma \sigma \sigma}(z)=z^{\frac{1+4 \Delta_{\sigma}}{3}}(1-z)^{-\Delta_{\sigma}} F_{1}\left(\frac{1-2 \Delta_{\sigma}}{3}, \frac{2+2 \Delta_{\sigma}}{3} ; \frac{4+4 \Delta_{\sigma}}{3} ; z\right) .
\end{aligned}
$$

First, let us look at the identity block. After a quadratic transformation, the hypergeometric function becomes a series in $\frac{z^{2}}{4 z-4}$. This makes the formula (2.9) applicable if we set $p=2 n$ and $q=n$.

$$
\begin{aligned}
G_{(1,1)}^{\sigma \sigma \sigma \sigma}(z) & ={ }_{2} F_{1}\left(-\Delta_{\sigma}, \frac{1+\Delta_{\sigma}}{3} ; \frac{5-4 \Delta_{\sigma}}{6} ; \frac{1}{4} \frac{z^{2}}{z-1}\right) \\
& =\sum_{n=0}^{\infty}\left(-\frac{1}{4}\right)^{n} \frac{\left(-\Delta_{\sigma}\right)_{n}\left(\frac{1+\Delta_{\sigma}}{3}\right)_{n}}{\left(\frac{5-4 \Delta_{\sigma}}{6}\right)_{n} n !} \sum_{m=0}^{\infty} \frac{(2 n)_{m}^{2}}{(4 n+m-1)_{m} m !}{ }^{3} F_{2}\left(\begin{array}{c}
-m, 4 n+m-1, n \\
2 n, 2 n
\end{array} ;\right) K_{2 n+m}(z) .
\end{aligned}
$$

\footnotetext{
${ }^{8}$ For the moment, we will be concerned with the global OPE coefficients contained within each one. The overall coefficients $C_{\left(r_{1}, s_{1}\right)\left(r_{2}, s_{2}\right)}^{\left(r_{3}, s_{3}\right)}$ are the generalized minimal model structure constants that were obtained with the Coulomb gas formalism in [38-40].
} 
Exchanging the two sums, we find

$$
c_{2 k}=\sum_{n=0}^{k}\left(-\frac{1}{4}\right)^{n} \frac{\left(-\Delta_{\sigma}\right)_{n}\left(\frac{1+\Delta_{\sigma}}{3}\right)_{n}}{\left(\frac{5-4 \Delta_{\sigma}}{6}\right)_{n} n !} \frac{(2 n)_{2 k-2 n}^{2}}{(2 n+2 k-1)_{2 k-2 n}(2 k-2 n) !}{ }^{3} F_{2}\left(\begin{array}{c}
2 n-2 k, 2 n+2 k-1, n \\
2 n, 2 n
\end{array} ;\right)
$$

for the nonvanishing global block coefficients. ${ }^{9}$ Since $p=2 q$, this hypergeometric is in a form that can be treated with Watson's theorem. When we apply this to the $c_{2 k}$, one pole in the numerator cancels another in the denominator, ${ }^{10}$

$$
\begin{aligned}
{ }_{3} F_{2}\left(\begin{array}{c}
2 n-2 k, 2 n+2 k-1, n \\
2 n, 2 n
\end{array} 1\right) & =\frac{(-1)^{n+k} \Gamma\left(\frac{1}{2}\right) \Gamma\left(n+\frac{1}{2}\right) \Gamma(2 n)}{\Gamma(n) \Gamma\left(n-k+\frac{1}{2}\right) \Gamma(2 n+2 k-1)} \frac{(k-1) !}{(n-1) !} \\
& =\frac{(-1)^{n+k} \Gamma(2 n)^{2}}{\Gamma(2 n+2 k-1)} \frac{\Gamma\left(n+k-\frac{1}{2}\right) \Gamma\left(\frac{1}{2}\right)}{\Gamma\left(n-k+\frac{1}{2}\right) \Gamma\left(k+\frac{1}{2}\right)} \lim _{\delta \rightarrow 0} \frac{2^{2 k-1}(k-1) !}{\left[\Gamma(\delta)(\delta)_{n}\right]^{2}} .
\end{aligned}
$$

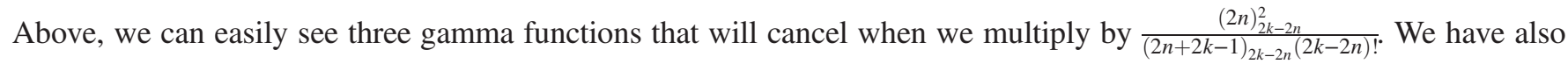
written $\frac{1}{\Gamma(n)^{2}}$ in a limiting form for later convenience. After substituting (3.9), one must use the identities

$$
\Gamma(x+n)=\Gamma(x)(x)_{n}, \quad \Gamma(x-n)=(-1)^{n} \frac{\Gamma(x)}{(1-x)_{n}}
$$

until each term of (3.8) only depends on $n$ through the Pochhammer symbol. This leads to

$$
\begin{aligned}
& c_{2 k}=\left(\begin{array}{c}
4 k-2 \\
2 k-1
\end{array}\right)^{-1} \lim _{\delta \rightarrow 0} \frac{1}{k(2 k-1) \Gamma(\delta)^{2}}{ }_{4} F_{3}\left(\begin{array}{c}
-k, k-\frac{1}{2},-\Delta_{\sigma}, \frac{1+\Delta_{\sigma}}{3} \\
\delta, \delta, \frac{5-4 \Delta_{\sigma}}{6}
\end{array}\right) \\
& =\left(\begin{array}{l}
4 k-2 \\
2 k-1
\end{array}\right)^{-1} \frac{\Delta_{\sigma}\left(1+\Delta_{\sigma}\right)}{5-4 \Delta_{\sigma}}{ }_{4} F_{3}\left(\begin{array}{c}
1-k, k+\frac{1}{2}, 1-\Delta_{\sigma}, \frac{4+\Delta_{\sigma}}{3} \\
1,2, \frac{11-4 \Delta_{\sigma}}{6}
\end{array} ;\right) \text {. }
\end{aligned}
$$

The $\epsilon$ block can be analyzed in the same way. Doing so will in fact be easier since we will not have to pass to the $\delta \rightarrow 0$ limit. Starting from

$$
G_{(1,3)}^{\sigma \sigma \sigma}(z)=z^{\frac{1+4 \Delta_{\sigma}}{3}}(1-z)^{-\frac{1+4 \Delta_{\sigma}}{6}}{ }_{2} F_{1}\left(\frac{1+2 \Delta_{\sigma}}{2}, \frac{1-2 \Delta_{\sigma}}{6} ; \frac{7+4 \Delta_{\sigma}}{6} ; \frac{1}{4} \frac{z^{2}}{z-1}\right),
$$

we may use (2.9) with $p=\frac{1+4 \Delta_{\sigma}}{3}+2 n$ and $q=\frac{1+4 \Delta_{\sigma}}{6}+n$. This leads to

$$
\begin{aligned}
c_{2 k}= & \sum_{n=0}^{k}\left(-\frac{1}{4}\right)^{n} \frac{\left(\frac{1+2 \Delta_{\sigma}}{6}\right)_{n}\left(\frac{1+2 \Delta_{\sigma}}{2}\right)_{n}}{\left(\frac{7+4 \Delta_{\sigma}}{6}\right)_{n} n !} \frac{\left(\frac{1+4 \Delta_{\sigma}}{3}+2 n\right)_{2 k-2 n}^{2}}{\left(\frac{2+8 \Delta_{\sigma}}{3}+2 n+2 k-1\right)_{2 k-2 n}(2 k-2 n) !} \\
& \times{ }_{3} F_{2}\left(\begin{array}{c}
2 n-2 k, \frac{2+8 \Delta_{\sigma}}{3}+2 n+2 k-1, \frac{1+4 \Delta_{\sigma}}{6}+n \\
\frac{1+4 \Delta_{\sigma}}{3}+2 n, \frac{1+4 \Delta_{\sigma}}{3}+2 n
\end{array}\right),
\end{aligned}
$$

which again allows us to use Watson's theorem. Employing (3.10) to perform the sum, we arrive at

$$
c_{2 k}=\frac{1}{4^{k} k !} \frac{\left(\frac{1+4 \Delta_{\sigma}}{6}\right)_{k}^{2}}{\left(\frac{1+4 \Delta_{\sigma}}{3}+k-\frac{1}{2}\right)_{k}}{ }_{4} F_{3}\left(\begin{array}{c}
-k, \frac{1+4 \Delta_{\sigma}}{3}+k-\frac{1}{2}, \Delta_{\sigma}+\frac{1}{2}, \frac{1-2 \Delta_{\sigma}}{6}, ; 1 \\
\frac{1+4 \Delta_{\sigma}}{6}, \frac{1+4 \Delta_{\sigma}}{6}, \frac{7+4 \Delta_{\sigma}}{6}
\end{array} ; .\right.
$$

A useful observation about the hypergeometric functions in (3.17) and (3.14) is that they both have a parameter excess of 1. This means that they are Wilson polynomials [30], ${ }^{11}$

\footnotetext{
${ }^{9}$ We derived this by applying a quadratic transformation to (3.6) which makes the Bose symmetry manifest. By leaving the function in its original form, or by applying Euler/Pfaff transformations, we can derive other sums that are nontrivially equivalent to (3.8).

${ }^{10}$ Because we have not allowed for other poles, our expression for $c_{2 k}$ will not be correct for $k=0$. There is no need to treat this case separately as it is already clear that $c_{0}=1$.

${ }^{11}$ Note that we are using the normalization in [41]. There is another common normalization that makes the Wilson polynomial symmetric in $(a, b, c, d)$.
} 


$$
P_{n}(a, b, c, d ; x)={ }_{4} F_{3}\left(\begin{array}{c}
-n, n+a+b+c+d-1, a+x, a-x \\
a+b, a+c, a+d
\end{array} ; 1\right)
$$

Invoking this notation, our results are

$$
\begin{aligned}
& c_{2 n}^{\sigma \sigma(1,1) \sigma \sigma}=\left(\begin{array}{c}
4 n-2 \\
2 n-1
\end{array}\right)^{-1} \frac{\Delta_{\sigma}\left(1+\Delta_{\sigma}\right)}{5-4 \Delta_{\sigma}} P_{n-1}\left(\frac{7-2 \Delta_{\sigma}}{6}, \frac{4-2 \Delta_{\sigma}}{6},-\frac{1-2 \Delta_{\sigma}}{6}, \frac{5+2 \Delta_{\sigma}}{6} ; \frac{1+4 \Delta_{\sigma}}{6}\right), \\
& c_{2 n}^{\sigma \sigma(1,3) \sigma \sigma}=\frac{1}{4^{n} n !} \frac{\left(\frac{1+4 \Delta_{\sigma}}{6}\right)_{n}^{2}}{\left(\frac{1+4 \Delta_{\sigma}}{3}+n-\frac{1}{2}\right)_{n}} P_{n}\left(\frac{2+2 \Delta_{\sigma}}{3}, \frac{1}{2},-\frac{1}{2},-\frac{1}{2} ; \frac{1+4 \Delta_{\sigma}}{6}\right) \text {. }
\end{aligned}
$$

As with continuous Hahn polynomials, there is a recurrence relation that the Wilson polynomials satisfy,

$$
\begin{aligned}
\left(x^{2}-a^{2}\right) P_{n}(x) & =A_{n} P_{n+1}(x)-\left(A_{n}+B_{n}\right) P_{n}(x)+B_{n} P_{n-1}(x), \\
A_{n} \equiv & \frac{(n+a+b+c+d-1)(n+a+b)(n+a+c)(n+a+d)}{(2 n+a+b+c+d-1)(2 n+a+b+c+d)}, \\
B_{n} \equiv & \frac{n(n+b+c-1)(n+b+d-1)(n+c+d-1)}{(2 n+a+b+c+d-2)(2 n+a+b+c+d-1)} .
\end{aligned}
$$

Appendix A uses this recursion to solve for the asymptotic behavior of Wilson polynomials and prove that the ones in (3.16) are positive.

\section{B. Some global block coefficients in the other correlators}

Given our success at explaining the one-correlator results, the next logical step is to find the global block decompositions applicable to three correlators. We will start with $\langle\epsilon \epsilon \epsilon \epsilon\rangle$. Although this is another four-point function of identical scalars, the main difference compared to $\langle\sigma \sigma \sigma \sigma\rangle$ is the lack of a closed-form Virasoro block. Because a generic $\epsilon$ only has a null descendant at level 3, we will have to work with a third-order BPZ equation which does not have simple solutions analogous to (3.6). This makes the formula (2.5) important for finding low-lying OPE coefficients. The singular vector that must annihilate

$$
\begin{aligned}
& \left\langle\epsilon\left(z_{1}, \bar{z}_{1}\right) \epsilon\left(z_{2}, \bar{z}_{2}\right) \epsilon\left(z_{3}, \bar{z}_{3}\right) \epsilon\left(z_{4}, \bar{z}_{4}\right)\right\rangle=\frac{G(z, \bar{z})}{\left|z_{12} z_{34}\right|^{2 \Delta_{\epsilon}}}, \\
& G(z, \bar{z})=G_{(1,1)}^{\epsilon \epsilon \epsilon \epsilon}(z) G_{(1,1)}^{\epsilon \epsilon \epsilon \epsilon}(\bar{z})+C_{(1,3)(1,3)}^{(1,3)} G_{(1,3)}^{\epsilon \epsilon \epsilon \epsilon}(z) G_{(1,3)}^{\epsilon \epsilon \epsilon \epsilon}(\bar{z})+C_{(1,3)(1,3)}^{(1,5)} G_{(1,5)}^{\epsilon \epsilon \epsilon}(z) G_{(1,5)}^{\epsilon \epsilon \epsilon}(\bar{z})
\end{aligned}
$$

has four terms that can be read off from (3.2). The Virasoro commutation relations reduce it to the three term expression

$$
\frac{4}{\Delta_{\epsilon}\left(\Delta_{\epsilon}+2\right)} \mathcal{L}_{-1}^{3}-\frac{4}{\Delta_{\epsilon}} \mathcal{L}_{-2} \mathcal{L}_{-1}+\mathcal{L}_{-3}
$$

The null state condition that follows from (3.18) and (3.19) is

$$
\begin{aligned}
& 4 z^{2}(z-1)^{3} \frac{\partial^{3} G}{\partial z^{3}}+4 z(z-1)^{2}\left[\left(4-\Delta_{\epsilon}\right) z+2 \Delta_{\epsilon}-2\right] \frac{\partial^{2} G}{\partial z^{2}} \\
& \quad-(z-1)\left[\left(\Delta_{\epsilon}^{2}+10 \Delta_{\epsilon}-8\right) z^{2}+\left(3 \Delta_{\epsilon}^{2}-14 \Delta_{\epsilon}+8\right) z-3 \Delta_{\epsilon}\left(\Delta_{\epsilon}-2\right)\right] \frac{\partial G}{\partial z} \\
& \quad+\Delta_{\epsilon}^{2}\left(\Delta_{\epsilon}+2\right) z(z-2) G=0 .
\end{aligned}
$$

To approximate the Virasoro blocks that solve this, it will be helpful to use the Frobenius method. ${ }^{12}$ Inserting the ansatz $G(z)=\sum_{k=-\infty}^{\infty} b_{k} z^{r+k}$, we may reindex the sum so that all terms carry the same power of $z$. This gives a recurrence relation for the coefficients.

\footnotetext{
${ }^{12}$ Even though they describe exchanged weights of $h_{1,1}$ and $h_{1,3}$, we cannot reuse either of the expressions in (3.6). Unlike global blocks which only see dimension differences, Virasoro blocks depend on the external weights individually [42].
} 


$$
\begin{aligned}
& {\left[-4(k+r)(k+r-1)+8\left(\Delta_{\epsilon}-1\right)(k+r)+3 \Delta_{\epsilon}\left(2-\Delta_{\epsilon}\right)\right](k+r+1) b_{k+1}} \\
& \quad+2\left[6(k+r-1)(k+r-2)+2\left(8-5 \Delta_{\epsilon}\right)(k+r-1)+3 \Delta_{\epsilon}^{2}-10 \Delta_{\epsilon}+4\right](k+r) b_{k} \\
& \quad-2\left[6(k+r-1)(k+r-2)(k+r-3)-4\left(2 \Delta_{\epsilon}-5\right)(k+r-1)(k+r-2)\right. \\
& \left.\quad+\left(\Delta_{\epsilon}^{2}-12 \Delta_{\epsilon}+8\right)(k+r-1)+\Delta_{\epsilon}^{2}\left(\Delta_{\epsilon}+2\right)\right] b_{k-1} \\
& \quad+\left[4(k+r-2)(k+r-3)(k+r-4)+4\left(4-\Delta_{\epsilon}\right)(k+r-2)(k+r-3)\right. \\
& \left.\quad-\left(\Delta_{\epsilon}^{2}+10 \Delta_{\epsilon}-8\right)(k+r-2)+\Delta_{\epsilon}^{2}\left(\Delta_{\epsilon}+2\right)\right] b_{k-2}=0 .
\end{aligned}
$$

We will set $b_{0}=1$ and $b_{k}=0$ for all $k<0$. The values of $r$ that make this consistent (called roots of the indicial equation) are $h_{1,1}, h_{1,3}$, and $h_{1,5}$ as expected. We find them by demanding that $b_{0}$ drop out of (3.21) when $k=-1$. For each value of $r$, it is straightforward to iterate (3.21) and then feed the results into (2.5). Some of the OPE coefficients that follow from this are written in Table III. Due to the appearance of the upper bound (1.4) in the three-correlator bootstrap, we expect the coefficients to be positive when $1 \leq \Delta_{\epsilon} \leq 2$, at least up to some high order. Figure 2 shows that this is indeed the case.

We should also be able to find positive squared OPE coefficients in the mixed correlator. The four-point function

$$
\begin{aligned}
& \left\langle\sigma\left(z_{1}, \bar{z}_{1}\right) \epsilon\left(z_{2}, \bar{z}_{2}\right) \sigma\left(z_{3}, \bar{z}_{3}\right) \epsilon\left(z_{4}, \bar{z}_{4}\right)\right\rangle=\left(\frac{\left|z_{24}\right|}{\left|z_{13}\right|}\right)^{\Delta_{\sigma \epsilon}} \frac{G(z, \bar{z})}{\left|z_{12}\right|^{\Delta_{\sigma}+\Delta_{\epsilon}}\left|z_{34}\right|^{\Delta_{\sigma}+\Delta_{\varepsilon}}}, \\
& G(z, \bar{z})=C_{(1,2)(1,3)}^{(1,2)} G_{(1,2)}^{\sigma \epsilon \sigma \epsilon}(z) G_{(1,2)}^{\sigma \epsilon \sigma \epsilon}(\bar{z})+C_{(1,2)(1,3)}^{(1,4)} G_{(1,4)}^{\sigma \sigma \epsilon}(z) G_{(1,4)}^{\sigma \epsilon \sigma}(\bar{z})
\end{aligned}
$$

satisfies second-order and third-order BPZ equations.

For simplicity, we will consider the second-order equation

$$
\begin{aligned}
& \frac{3}{2} z^{2}(z-1)^{2} \frac{\partial^{2} G}{\partial z^{2}}+\frac{1}{2} z(z-1)\left[\left(2-7 \Delta_{\sigma}\right) z+9 \Delta_{\sigma}\right] \frac{\partial G}{\partial z} \\
& \quad+\frac{1}{24}\left[3 \Delta_{\sigma}\left(11 \Delta_{\sigma}+2\right) z^{2}-2\left(5 \Delta_{\sigma}+2\right)\left(11 \Delta_{\sigma}+2\right) z+9 \Delta_{\sigma}\left(5 \Delta_{\sigma}+2\right)\right] G=0
\end{aligned}
$$

which has the recurrence relation

$$
\begin{aligned}
& 9\left[4(k+r+1)(k+r)-12 \Delta_{\sigma}(k+r+1)+\Delta_{\sigma}\left(5 \Delta_{\sigma}+2\right)\right] b_{k+1} \\
& \quad-2\left[36(k+r)(k+r-1)-12\left(8 \Delta_{\sigma}-1\right)(k+r)+\left(5 \Delta_{\sigma}+2\right)\left(11 \Delta_{\sigma}+2\right)\right] b_{k} \\
& \quad+3\left[12(k+r-1)(k+r-2)+4\left(2-7 \Delta_{\sigma}\right)(k+r-1)+\Delta_{\sigma}\left(11 \Delta_{\sigma}+2\right)\right] b_{k-1}=0 .
\end{aligned}
$$

It is easily seen that $r \in\left\{h_{1,2}, h_{1,4}\right\}$ is the solution of the indicial equation for (3.32). Because the product $\sigma \times \epsilon$ no longer has Bose symmetry, the procedure by which we extract the conformal block expansion this time is somewhat different. We must include dimension differences in the hypergeometric function and sum over all integers whether even or odd,

$$
\begin{aligned}
G(z) & =\sum_{n=0}^{\infty}(-1)^{n} c_{n} z^{r+n}{ }_{2} F_{1}\left(r+n-\frac{1}{2} \Delta_{\sigma \epsilon}, r+n+\frac{1}{2} \Delta_{\sigma \epsilon} ; 2(r+n) ; z\right) \\
& =\sum_{n=0}^{\infty} \sum_{m=0}^{\infty}(-1)^{n} c_{n} \frac{\left(r+n-\frac{1}{2} \Delta_{\sigma \epsilon}\right)_{m}\left(r+n+\frac{1}{2} \Delta_{\sigma \epsilon}\right)_{m}}{(2 r+2 n)_{m}} \frac{z^{r+n+m}}{m !} \\
& =\sum_{k=0}^{\infty} \sum_{n=0}^{k}(-1)^{n} c_{n} \frac{\left(r+n-\frac{1}{2} \Delta_{\sigma \epsilon}\right)_{k-n}\left(r+n+\frac{1}{2} \Delta_{\sigma \epsilon}\right)_{k-n}}{(2 r+2 n)_{k-n}} \frac{z^{r+k}}{(k-n) !} .
\end{aligned}
$$

The lower triangular system from this leads to the recursion

$$
(-1)^{k} c_{k}=b_{k}-\sum_{n=0}^{k-1}(-1)^{n} c_{n} \frac{\left(r+n-\frac{1}{2} \Delta_{\sigma \epsilon}\right)_{k-n}\left(r+n+\frac{1}{2} \Delta_{\sigma \epsilon}\right)_{k-n}}{(2 r+2 n)_{k-n}(k-n) !}
$$

Some low-lying global block coefficients found with (3.26) are listed in Table IV. While all of them are non-negative above the tricritical Ising value $\Delta_{\sigma}=\frac{1}{5}$, there is actually one that takes on negative values for $\frac{1}{8}<\Delta_{\sigma}<\frac{1}{5}$ as shown in Fig. 3. We 
TABLE III. The first three global block coefficients in the $(1,1)$, $(1,3)$, and $(1,5)$ contributions found by taking the $\epsilon \times \epsilon$ OPE twice.

\begin{tabular}{ll}
\hline \hline$n$ & \multicolumn{1}{c}{$c_{2 n}^{\epsilon \epsilon(1,1) \epsilon \epsilon}$} \\
\hline 0 & 1 \\
1 & $-\frac{\Delta_{\epsilon}^{2}\left(\Delta_{\epsilon}+2\right)}{\left(\Delta_{\epsilon}-4\right)\left(3 \Delta_{\epsilon}-2\right)}$ \\
2 & $\frac{\Delta_{\epsilon}^{2}\left(\Delta_{\epsilon}+2\right)^{2}\left[5 \Delta_{\epsilon}+2\right]}{30\left(\Delta_{\epsilon}-8\right)\left(\Delta_{\epsilon}-4\right)\left(3 \Delta_{\epsilon}-2\right)}$ \\
$n$ & $c_{2 n}^{\epsilon \epsilon(3,1) \epsilon \epsilon}$ \\
0 & 1 \\
1 & $\frac{\Delta_{\epsilon}^{2}\left(\Delta_{\epsilon}+2\right)\left[5 \Delta_{\epsilon}+2\right]}{16\left(\Delta_{\epsilon}-1\right)\left(\Delta_{\epsilon}+1\right)\left(\Delta_{\epsilon}+4\right)}$ \\
2 & $\frac{\Delta_{\epsilon}^{2}\left(\Delta_{\epsilon}+2\right)\left[25 \Delta_{\epsilon}^{5}+167 \Delta_{\epsilon}^{4}-66 \Delta_{\epsilon}^{3}-1904 \Delta_{\epsilon}^{2}-2752 \Delta_{\epsilon}-384\right]}{512\left(\Delta_{\epsilon}-3\right)\left(\Delta_{\epsilon}-1\right)\left(\Delta_{\epsilon}+3\right)\left(\Delta_{\epsilon}+4\right)\left(\Delta_{\epsilon}+5\right)\left(\Delta_{\epsilon}+8\right)}$ \\
$n$ & $c_{2 n}^{\epsilon \epsilon(5,1) \epsilon \epsilon}$ \\
0 & 1 \\
1 & $\frac{\Delta_{\epsilon}\left(\Delta_{\epsilon}+2\right)\left[7 \Delta_{\epsilon}+6\right]}{48\left(\Delta_{\epsilon}+1\right)\left(\Delta_{\epsilon}+3\right)}$ \\
2 & $\frac{\Delta_{\epsilon}\left(\Delta_{\epsilon}+2\right)\left[441 \Delta_{\epsilon}^{5}+5121 \Delta_{\epsilon}^{4}+20732 \Delta_{\epsilon}^{3}+37796 \Delta_{\epsilon}^{2}+31056 \Delta_{\epsilon}+8640\right]}{1536\left(\Delta_{\epsilon}+3\right)\left(\Delta_{\epsilon}+5\right)\left(3 \Delta_{\epsilon}+5\right)\left(3 \Delta_{\epsilon}+7\right)\left(3 \Delta_{\epsilon}+10\right)}$ \\
\hline \hline
\end{tabular}

TABLE IV. The first five global block coefficients in the $(1,2)$ and $(1,4)$ contributions found by taking the $\sigma \times \epsilon$ OPE twice.

\begin{tabular}{ll}
\hline \hline$n$ & \multicolumn{1}{c}{$c_{n}^{\sigma \epsilon(1,2) \sigma \epsilon}$} \\
\hline 0 & 1 \\
1 & 0 \\
2 & 0 \\
3 & $-\frac{\left(\Delta_{\sigma}+1\right)\left(4 \Delta_{\sigma}+1\right)^{2}}{729 \Delta_{\sigma}\left(\Delta_{\sigma}-1\right)\left(\Delta_{\sigma}+2\right)}\left(5 \Delta_{\sigma}+2\right)$ \\
4 & $-\frac{4\left(\Delta_{\sigma}+1\right)^{2}\left(4 \Delta_{\sigma}+1\right)^{2}}{729 \Delta_{\sigma}\left(\Delta_{\sigma}+3\right)\left(\Delta_{\sigma}+6\right)\left(2 \Delta_{\sigma}-3\right)}\left(8 \Delta_{\sigma}-1\right)$ \\
$n$ & $c_{n}^{\sigma \epsilon(1,4) \sigma \epsilon}$ \\
0 & 1 \\
1 & 0 \\
2 & $\frac{\Delta_{\sigma}+1}{9\left(2 \Delta_{\sigma}+3\right)\left(5 \Delta_{\sigma}+3\right)}\left(2 \Delta_{\sigma}+1\right)\left(10 \Delta_{\sigma}+1\right)$ \\
3 & $\frac{4\left(\Delta_{\sigma}+1\right)\left(5 \Delta_{\sigma}+2\right)}{729\left(\Delta_{\sigma}+2\right)\left(5 \Delta_{\sigma}+4\right)\left(5 \Delta_{\sigma}+6\right)}\left(5 \Delta_{\sigma}-1\right)\left(7 \Delta_{\sigma}+4\right)$ \\
4 & $\frac{\left(\Delta_{\sigma}+1\right)^{2}\left(5 \Delta_{\sigma}+2\right)}{81\left(5 \Delta_{\sigma}+7\right)\left(5 \Delta_{\sigma}+8\right)}\left(10 \Delta_{\sigma}+1\right)$ \\
\hline \hline
\end{tabular}

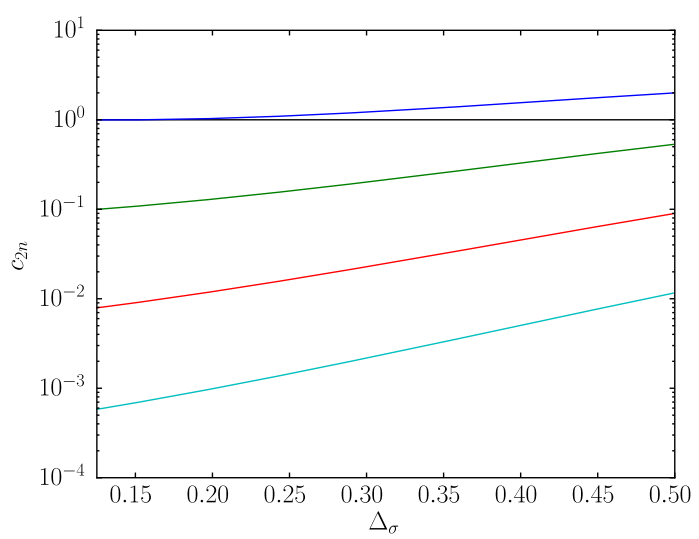

(a) $c_{2 n}^{\epsilon \epsilon(1,1) \epsilon \epsilon}$

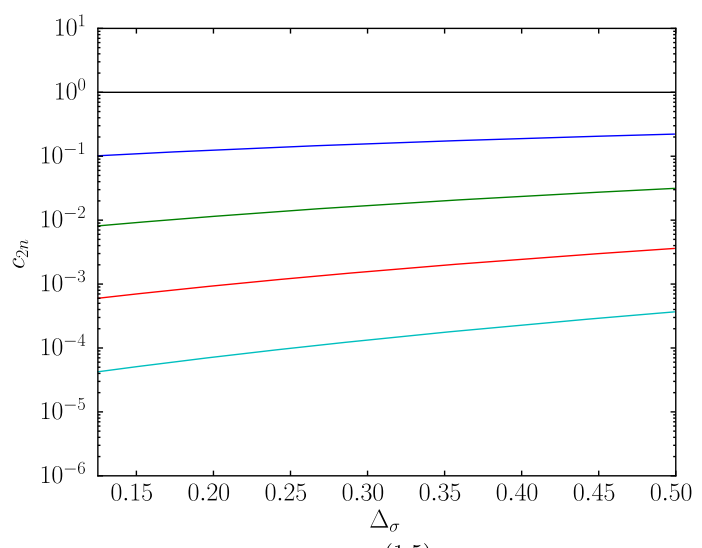

(c) $c_{2 n}^{\epsilon \epsilon(1,5) \epsilon \epsilon}$

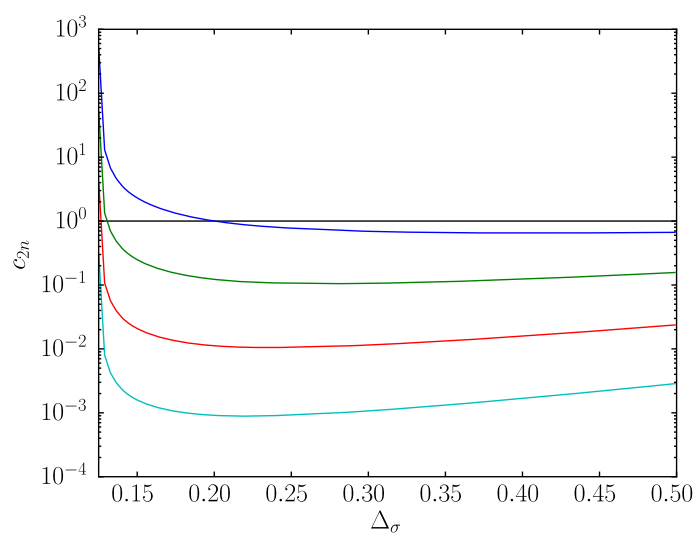

(b) $c_{2 n}^{\epsilon \epsilon(1,3) \epsilon \epsilon}$

FIG. 2. Log-scale plots of $c_{2 n}^{\epsilon \epsilon(1, s) \epsilon \epsilon}$ showing that the first five are all positive on the interval $\frac{1}{8} \leq \Delta_{\sigma} \leq \frac{1}{2}$. 


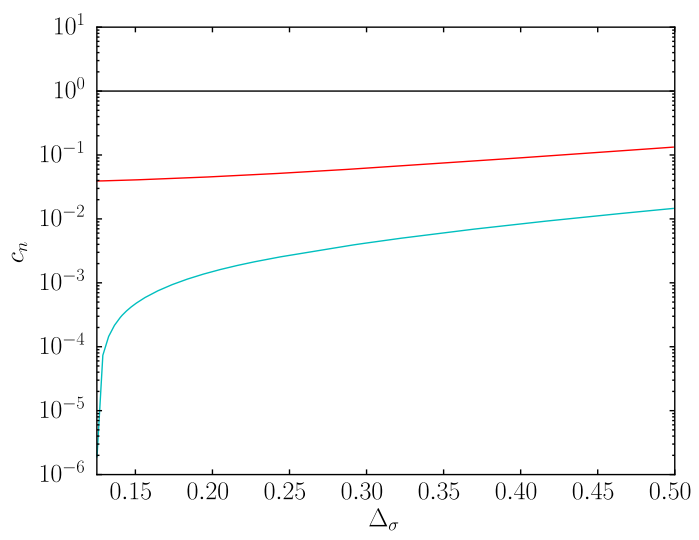

(a) $c_{n}^{\sigma \epsilon(1,2) \sigma \epsilon}$

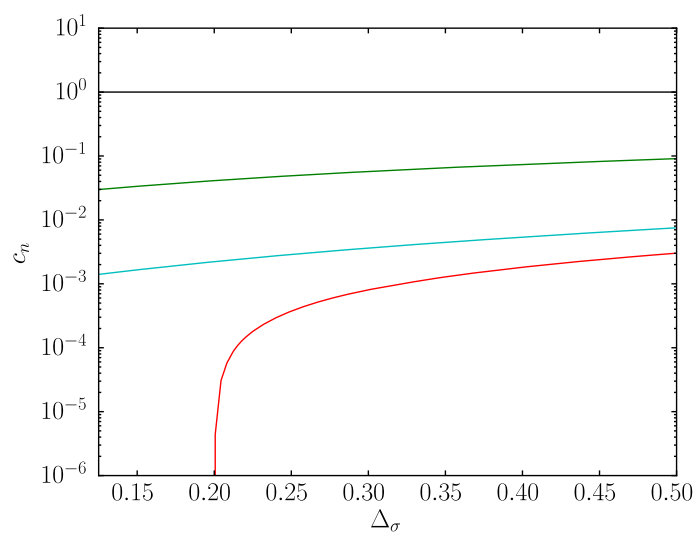

(b) $c_{n}^{\sigma \epsilon(1,4) \sigma \epsilon}$

FIG. 3. Log-scale plots of the $c_{n}^{\sigma \epsilon(1, s) \sigma \epsilon}$ that are nonzero. The legend is the same as that of Fig. 2.

may explain this by noticing that $\phi_{1,4}$ is also $\phi_{3,1}$ in the minimal model $\mathcal{M}(5,4)$. This field has exactly one quasiprimary descendant at level 3 . The presence of a null state is therefore enough to conclude that $c_{3}^{\sigma \epsilon(1,4) \sigma \epsilon}=0$.

Alternatively, we can take the OPE in the other channel by permuting operator positions in (3.22). This yields the global block coefficients in Table $\mathrm{V}$ which are related to the $\lambda_{\sigma \sigma \mathcal{O}} \lambda_{\epsilon \epsilon \mathcal{O}}$ CFT data. There is no reason for these numbers to be positive, but we expect

$$
c_{2 n}^{\sigma \sigma(1, s) \sigma \sigma} c_{2 n}^{\epsilon \epsilon(1, s) \epsilon \epsilon} \geq\left(c_{2 n}^{\sigma \sigma(1, s) \epsilon \epsilon}\right)^{2}
$$

to be obeyed. ${ }^{13}$ When $\sigma \times \sigma$ and $\epsilon \times \epsilon$ share a set of operators $\mathcal{S}$ with the same quantum numbers, the left-hand side is a product of sums. The right-hand side is a sum of products and therefore smaller by the arithmetic-geometric mean inequality. A departure from (3.27) would be a violation of unitarity since the matrix

$$
\sum_{\mathcal{O} \in \mathcal{S}}\left[\begin{array}{ll}
\lambda_{\sigma \sigma \mathcal{O}} & \lambda_{\epsilon \epsilon \mathcal{O}}
\end{array}\right]\left[\begin{array}{l}
\lambda_{\sigma \sigma \mathcal{O}} \\
\lambda_{\epsilon \epsilon \mathcal{O}}
\end{array}\right]=\left[\begin{array}{cc}
c_{2 n}^{\sigma \sigma(1, s) \sigma \sigma} & c_{2 n}^{\sigma \sigma(1, s) \epsilon \epsilon} \\
c_{2 n}^{\sigma \sigma(1, s) \epsilon \epsilon} & c_{2 n}^{\epsilon \epsilon(1, s) \epsilon \epsilon}
\end{array}\right]
$$

would not be positive definite.

\section{Virasoro block coefficients}

Our analysis so far has been focused on $c_{2 n}^{\sigma \sigma(1, s) \sigma \sigma}$, $c_{2 n}^{\epsilon \epsilon(1, s) \epsilon \epsilon}$, and $c_{n}^{\sigma \epsilon(1, s) \sigma \epsilon}$, which encode the decomposition of a Virasoro block into $\mathfrak{I}(2)$ blocks. With the sole exception of $c_{3}^{\sigma \epsilon(1,4) \sigma \epsilon}$, which we could imagine to have a small effect, we have found that these coefficients are non-negative when $\frac{1}{8} \leq \Delta_{\sigma} \leq \frac{1}{2}$. However, this is only

\footnotetext{
${ }^{13}$ For another quick check of our results, $\Delta_{\epsilon}^{2} c_{0}^{\sigma \sigma(1,1) \sigma \sigma} c_{2}^{\sigma \sigma(1,1) \sigma \sigma}=$ $\Delta_{\sigma}^{2} c_{0}^{\epsilon \epsilon(1,1) \epsilon \epsilon} c_{2}^{\epsilon \epsilon(1,1) \epsilon \epsilon}$ holds as it must by the Ward identity.
}

meaningful if the same property holds for the structure constants that unite holomorphic and antiholomorphic halves of a four-point function. A single $C_{\left(r_{1}, s_{1}\right)\left(r_{2}, s_{2}\right)}^{\left(r_{3}, s_{3}\right)}<0$ for instance would give rise to an infinite number of negative contributions in $\left\langle\phi_{r_{1}, s_{1}} \phi_{r_{2}, s_{2}} \phi_{r_{1}, s_{1}} \phi_{r_{2}, s_{2}}\right\rangle$, severely complicating the interpretation of Fig. 1.

In a given correlation function, the Virasoro block coefficients that appear must be compatible with crossing symmetry and single valuedness. By briefly reviewing the method of [38-40], we will show that this condition is enough to fix them uniquely. For definiteness, consider the $\langle\epsilon \epsilon \epsilon \epsilon\rangle$ correlator in the generalized minimal model with central charge $\frac{13}{21}$. The holomorphic part of this function comes from the kernel of the operator (3.19), which is three dimensional. Solving (3.21) has given us a basis for this kernel in which each function vanishes at $z=0$. We could equally well have chosen any of the regular singular points 0,1 , and $\infty$, corresponding to the $s, t$, and $u$ channels for Virasoro blocks. When going from $z=0$ to $z=1$, there is a special matrix $F$ called the crossing matrix (or fusion matrix) that accomplishes $G^{a}(z)=F^{a}{ }_{b} G^{b}(1-z)$. It is a special case of the crossing kernel which applies to theories with a

TABLE V. The first three global block coefficients in the $(1,1)$ and $(1,3)$ contributions found by taking the $\sigma \times \sigma$ and $\epsilon \times \epsilon$ OPEs.

\begin{tabular}{ll}
\hline \hline$n$ & \multicolumn{1}{c}{$c_{2 n}^{\sigma \sigma(1,1) \epsilon \epsilon}$} \\
\hline 0 & 1 \\
1 & $\frac{\left(\Delta_{\sigma}+1\right)\left(4 \Delta_{\sigma}+1\right)}{3\left(5-4 \Delta_{\sigma}\right)}$ \\
2 & $\frac{2\left(\Delta_{\sigma}+1\right)^{2}\left(4 \Delta_{\sigma}+1\right)\left[5 \Delta_{\sigma}+2\right]}{45\left(5-4 \Delta_{\sigma}\right)\left(11-4 \Delta_{\sigma}\right)}$ \\
$n$ & $c_{2 n}^{\sigma \sigma(1,3) \epsilon \epsilon}$ \\
0 & 1 \\
1 & $\frac{2\left(\Delta_{\sigma}+1\right)\left(4 \Delta_{\sigma}+1\right)\left[5 \Delta_{\sigma}+2\right]}{3\left(7+4 \Delta_{\sigma}\right)\left(5+8 \Delta_{\sigma}\right)}$ \\
2 & $\frac{\left(\Delta_{\sigma}+1\right)^{2}\left(4 \Delta_{\sigma}+1\right)\left[400 \Delta_{\sigma}^{3}+1548 \Delta_{\sigma}^{2}+1644 \Delta_{\sigma}+361\right]}{18\left(7+4 \Delta_{\sigma}\right)\left(13+4 \Delta_{\sigma}\right)\left(11+8 \Delta_{\sigma}\right)\left(17+8 \Delta_{\sigma}\right)}$ \\
\hline \hline
\end{tabular}


continuous spectrum. ${ }^{14}$ Since it represents a change of basis, the expression for $F$ is unique. In this case, it is given by

$$
F=\left[\begin{array}{ccc}
0.7422 & 0.3124 & -0.1563 \\
2.3762 & -1.8795 & 1.4405 \\
1.8760 & -2.2733 & 2.1372
\end{array}\right]
$$

which was obtained in [43] via the Coulomb gas formalism. Later reviews are $[23,44]$. In the following, we will use $G^{1}$, $G^{2}$, and $G^{3}$ to denote $G_{(1,1)}^{\epsilon \epsilon \epsilon}, G_{(1,3)}^{\epsilon \epsilon \epsilon \epsilon}$, and $G_{(1,5)}^{\epsilon \epsilon \epsilon}$, respectively.

The constraints from (3.29) are best phrased in terms of a metric on the space of conformal blocks; $G(z, \bar{z})=W_{a b} G^{a}(z) G^{b}(\bar{z})$. We have three conditions that $W_{a b}$ must satisfy:

(1) $W_{11}=1$

(2) $W_{c d}=W_{a b} F_{c}^{a} F^{b}{ }_{d}$

(3) $W_{a b}=0$ for $a \neq b$

The first of these is an obvious consequence of having unitnormalized operators. The second comes from writing $G(1-z, 1-\bar{z})=W_{a b} G^{a}(1-z) G^{b}(1-\bar{z})$ in terms of $s-$ channel blocks and setting it equal to $G(z, \bar{z})$. The third ensures that the four-point function has trivial monodromy under $z \mapsto e^{2 \pi i} z, \bar{z} \mapsto e^{-2 \pi i} \bar{z}$. This rules out a nondiagonal metric since we only find $h_{r, s}$ weights that differ by integers at special values of $m .{ }^{15}$ We build solutions out of the left eigenvectors of $F$,

$v^{1}=\left[\begin{array}{c}0.9537 \\ -0.1157 \\ 0.2776\end{array}\right], v^{2}=\left[\begin{array}{c}0.9805 \\ 0.1758 \\ -0.0879\end{array}\right], v^{3}=\left[\begin{array}{c}0.5938 \\ -0.7196 \\ 0.3600\end{array}\right]$,

which have eigenvalues of 1,1 , and -1 , respectively. Clearly $F^{2}=1$, which follows from $z \leftrightarrow 1-z$ being an involution, requires all eigenvalues to be \pm 1 . The following form is invariant under two multiplications by $F$ :

$W_{a b}=c_{11} v_{a}^{1} v_{b}^{1}+c_{12} v_{a}^{1} v_{b}^{2}+c_{21} v_{a}^{2} v_{b}^{1}+c_{22} v_{a}^{2} v_{b}^{2}+c_{33} v_{a}^{3} v_{b}^{3}$.

There are six off-diagonal components of $W$ that need to vanish. If we set $c_{21}=c_{12}$, Eq. (3.31) becomes manifestly symmetric and we can use three more parameters, $c_{11}, c_{12}$, and $c_{22}$, to make $W$ diagonal. As the single remaining

\footnotetext{
${ }^{14}$ Several interchangeable terms have proliferated over the years. When replacing the $z \mapsto 1-z$ map with $z \mapsto \frac{1}{z}$, the words crossing and fusion become exchange and braiding. Outside the CFT context, one says that a linear ODE has a monodromy matrix or connection matrix. For ODEs that have less structure than a BPZ equation, finding this matrix is often a difficult problem.

${ }^{15}$ It is well known that this happens in discrete minimal models. Some of the nondiagonal theories so constructed also satisfy the stronger requirement of modular invariance.
}

parameter, $c_{33}$ is used to rescale $W$ so that its leading component is 1 .

We may summarize this discussion by stating that there is no freedom in the three-correlator bootstrap equations once the Virasoro blocks involving $\sigma$ and $\epsilon$ are specified. Knowledge of these blocks fully determines $\langle\sigma \sigma \sigma \sigma\rangle$, $\langle\sigma \sigma \epsilon \epsilon\rangle$, and $\langle\epsilon \epsilon \epsilon \epsilon\rangle$, whether or not we demand consistency conditions for other correlators. This means that the generalized minimal model structure constants are the only valid choices for the coefficients in (3.3), (3.25), and (3.22). Defining $\gamma(x)=\Gamma(x) / \Gamma(1-x)$ and $t=\frac{m}{m+1}$, the expressions we need are

$$
\begin{aligned}
C_{(1,3)(1,3)}^{(1,3)}= & \gamma(t)^{3} \gamma(4 t-1)^{2} \gamma(1-2 t)^{3} \gamma(2-2 t) \gamma(2-3 t), \\
C_{(1,3)(1,3)}^{(1,5)}= & \gamma(t) \gamma(2 t) \gamma(4 t-1) \gamma(5 t-1) \gamma(1-3 t) \gamma(1-4 t) \\
& \times \gamma(2-2 t) \gamma(2-3 t), \\
C_{(1,2)(1,3)}^{(1,4)}= & \gamma(t) \gamma(4 t-1) \gamma(1-3 t) \gamma(2-2 t), \\
C_{(1,2)(1,3)}^{(1,2)}= & C_{(1,2)(1,2)}^{(1,3)}=\gamma(t) \gamma(3 t-1) \gamma(1-2 t) \gamma(2-2 t) .
\end{aligned}
$$

The last coefficient in (3.32) is clearly the one that was rederived in [17]. Plotting these in Fig. 4, we see that $C_{(1,3)(1,3)}^{(1,5)}<0$ for the generalized minimal models between $\mathcal{M}(4,3)$ and $\mathcal{M}(5,4)$. This reveals a problem with our strategy for proving that the allowed region in Fig. 1 must be large enough to include (1.4). Constructing the generalized minimal model solution to crossing symmetry only accomplishes this in the one-correlator case. We must therefore conclude that there is at least one other way to extend the unitary subsector $\langle\sigma \sigma \sigma \sigma\rangle$ into a consistent threecorrelator system. This solution to crossing should have positive squared OPE coefficients wherever it exists, not

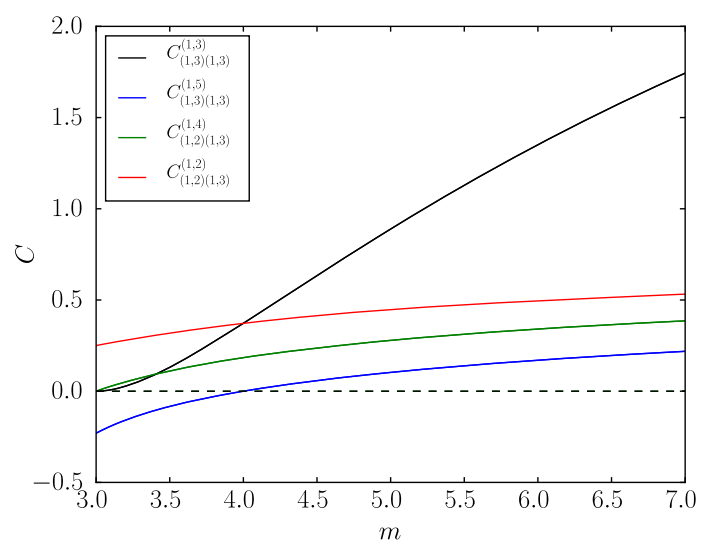

FIG. 4. Low-lying squared OPE coefficients for Virasoro primaries in the generalized minimal models. Between the Ising model at $m=3\left(\Delta_{\sigma}=\frac{1}{8}\right)$ and the tricritical Ising model at $m=4$ $\left(\Delta_{\sigma}=\frac{1}{5}\right)$, there is one that becomes negative. 
just in $\frac{1}{5} \leq \Delta_{\sigma} \leq \frac{1}{2}$. As the solution might be very different from the theories discussed above, it is worth using the numerical bootstrap to see what else can be learned about it.

\section{LESSONS FOR THE BOOTSTRAP}

We saw in the last section that above central charge $\frac{7}{10}$, the generalized minimal models exhibit the restricted notion of unitarity that allows them to appear in Fig. 1. On the other hand, for $\frac{1}{2}<c<\frac{7}{10}$, they are highly nonunitary at the level of three correlators; the global coefficient $c_{3}^{\sigma \epsilon(1,4) \sigma \epsilon}$ and the Virasoro coefficient $C_{(1,3)(1,3)}^{(1,5)}$ both become negative in this region. Working around this problem, the machinery of the bootstrap has filled in this region with another solution whose $\sigma \times \sigma$ OPE agrees with that of a generalized minimal model. In this section, we will give an intuitive argument for why this should be possible. Beyond this, we will discuss two issues related to the replacement solution.

This first is whether it can be found uniquely. A technique called the extremal functional method is often used to extract a unique solution to crossing symmetry and unitarity whenever a dimension gap or OPE coefficient is extremized [29,45-50]. Based on this, we might expect to find a single line of exotic solutions that smoothly joins the $\mathcal{M}(m+1, m)$ line at $\Delta_{\sigma}=\frac{1}{5}$. We will actually find the opposite-a boundary of Fig. 1 that has many possible choices for the local CFT data outside $\sigma \times \sigma$. To reconcile this with the standard lore about extremality, one has to remember that the bootstrap equations take on a more intricate form when there are multiple correlators.

The second is the prospect of excluding the above solution with further numerics. One reason for doing this with global conformal blocks is simply the technical challenge posed by Virasoro conformal blocks. There has indeed been recent progress in using the full Virasoro symmetry to carve out $c>1$ CFTs [51]. However, tractable four-point functions with extended supersymmetry appear to be limited to those of BPS operators [52,53]. Global blocks were therefore a necessary ingredient of [54], a program which aims to constrain the space of superconformal theories using external operators in long multiplets. There has also been recent interest in conformal theories that have no locality and therefore no Virasoro algebra [55-57]. These provide a different motivation for shrinking the regions in Fig. 1.

\section{A. Reduction to one correlator}

The well known bootstrap constraints for three correlators with $\mathbb{Z}_{2}$ symmetry take the form of five crossing equations. As reviewed in Appendix B, the vector of equations has one component for $\langle\sigma \sigma \sigma \sigma\rangle$, one component for $\langle\epsilon \epsilon \epsilon \epsilon\rangle$, and three components for $\langle\sigma \sigma \epsilon \epsilon\rangle$. Given a generic solution to crossing, it is easy to see that four (three) sum rules will break when an even (odd) operator is removed from the theory. However, there is a pleasing nongeneric property that holds for generalized minimal models; $\epsilon \times \epsilon$ contains more operators than $\sigma \times \sigma$. Because of this, only one crossing equation is disturbed when we remove the $\phi_{1,5}$ conformal family. This is the source of almost all unitarity violation in the system built from $\phi_{1,2}$ and $\phi_{1,3}$. Since negativity of $c_{3}^{\sigma \epsilon(1,4) \sigma \epsilon}$ only affects spinning operators with $\Delta>\frac{45}{8}$, it is possible that the numerics are largely insensitive to it [58]. Assuming that problems with the mixed correlator are negligible, we will focus on

$$
\begin{aligned}
\sum_{\mathcal{O}} \lambda_{\epsilon \epsilon \mathcal{O}}^{2} F_{-, \mathcal{O}}^{\epsilon \epsilon ; \epsilon \epsilon}(u, v) & =0, \\
F_{-, \Delta, \ell}^{\epsilon \epsilon ; \epsilon \epsilon}(u, v) & \equiv v^{\Delta_{\epsilon}} g_{\Delta, \ell}^{0,0}(u, v)-u^{\Delta^{{ }_{e}}} g_{\Delta, \ell}^{0,0}(v, u)
\end{aligned}
$$

as the single condition that needs to be restored. Once a solution to (4.1) is found, one can incorporate it into the three-correlator problem by choosing $\lambda_{\sigma \sigma \mathcal{O}}=0$ for new operators. $^{16}$

Checking the solvability of (4.1) for real $\lambda_{\epsilon \epsilon \mathcal{O}}$ is the simplest numerical bootstrap problem. Emphasizing the contributions of operators that are already present, we may write

$$
\begin{aligned}
\sum_{\mathcal{O}} \lambda_{\epsilon \epsilon \mathcal{O}}^{2} F_{-, \mathcal{O}}^{\epsilon \epsilon \epsilon \epsilon}(u, v) & =-F_{1,1}(u, v)-C_{(1,3)(1,3)}^{(1,3)} F_{1,3}(u, v), \\
F_{1,1}(u, v) \equiv & \sum_{n, \bar{n}} c_{n}^{\epsilon \epsilon(1,1) \epsilon \epsilon} c_{\bar{n}}^{\epsilon \epsilon(1,1) \epsilon \epsilon} F_{-, n+\bar{n},|n-\bar{n}|}^{\epsilon \epsilon ; \epsilon}(u, v) \\
F_{1,3}(u, v) \equiv & \sum_{n, \bar{n}} c_{n}^{\epsilon \epsilon(1,3) \epsilon \epsilon} c_{\bar{n}}^{\epsilon \epsilon(1,3) \epsilon \epsilon} \\
& \times F_{-, \Delta_{e}+n+\bar{n},|n-\bar{n}|}^{\epsilon \epsilon ; \epsilon \epsilon}(u, v) .
\end{aligned}
$$

Keeping operators with $\Delta \leq 30$, we have used the results of the last section to approximate the right-hand side of (4.2). In the sum over operators, there will be a continuum of irrelevant scalars not in $\phi_{1,1} \cup \phi_{1,3}$ which begins at some gap $\Delta_{*}>2$. If this is the only set of scalars on the left-hand side of (4.2), we are dealing with the set $\mathcal{S}=\left\{\Delta>\Delta_{*}\right\}$. Alternatively, we could allow the dimension $\Delta_{\epsilon}$ to appear again and enlarge it to $\mathcal{S}=\left\{\Delta>\Delta_{*}\right\} \cup\left\{\Delta=\Delta_{\epsilon}\right\}$. The second choice is the one applicable to Fig. 1. However, in Fig. 5, we consider the first choice as well. This is because it is possible to rederive Fig. 1 under the requirement that $\epsilon$ is nondegenerate [16]. As numerical accuracy is improved,

\footnotetext{
${ }^{16}$ We are using four crossing equations to derive (1.4) analytically and then claiming that the fifth crossing equation can be satisfied for free. This is different from what happens in three dimensions. We have checked that the island in [14] merges with the rest of the allowed region once the fifth crossing equation is dropped.
} 


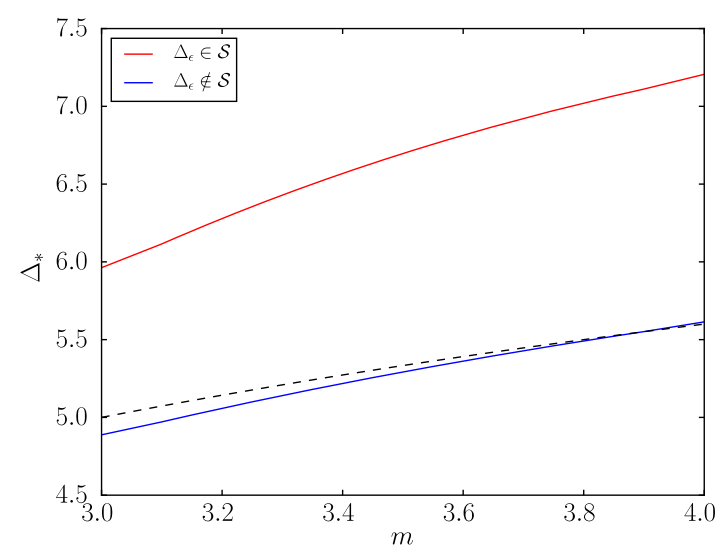

FIG. 5. Dimension bounds for irrelevant operators in (4.2). The dotted line shows the dimension of the primary scalar $\phi_{1,5}$ whose multiplet needs to be replaced for $3<m<4$.

we expect the blue line to precisely meet the dotted line at $m=4 .^{17}$

In Fig. 5, the red curve tells us that (1.4) is admissible whenever we treat $\left(\Delta_{\sigma}, \Delta_{\epsilon}\right)$ as allowed dimensions and perform a two-parameter scan. The blue curve tells us that (1.4) will still be admissible when we fix $\epsilon$ as a single operator at angle $\theta=\arctan \left(\frac{\lambda_{\sigma \sigma \epsilon}}{\lambda_{c e \epsilon}}\right)$ and scan over $\left(\Delta_{\sigma}, \Delta_{\epsilon}, \theta\right)$. Finally, one may contemplate the effect of imposing $\theta=\frac{\pi}{2}$ which is one consequence of Kramers-Wannier duality in the Ising model. Although this question cannot be answered with Fig. 5, we have found that (1.4) persists yet again. In all three cases, Eq. (1.4) is not just an allowed line-it is the maximal allowed line. From this, we must conclude that many solutions to crossing, labeled by values of $\Delta_{*}$ between the red and blue lines, lie along the bound on the left side of Fig. 1. This signals the presence of a flat direction, e.g. a bound in $\left(\Delta_{\sigma}, \Delta_{\epsilon}, \theta\right)$ space which is independent of $\theta$. A flat direction in the modular bootstrap was previously seen in [59]. The main argument for unique extremal functionals comes from [46], in which the multicorrelator bootstrap equations were augmented with angles for each operator in OPE space. To extract a spectrum in this formulation, one would have to look for zeros of these functionals on the entire $(\Delta, \theta)$ plane. We suspect that the flat direction here corresponds to a zero being achieved on a codimension-one locus.

Because the generalized minimal model line (1.4) is allowed by the bootstrap, there are several lines in the interior of Fig. 1 that must be allowed as well. These can be constructed through one or more tensor products. If we multiply two generalized minimal models for instance, the only nontrivial operator whose dimension lies to the left of $\Delta_{\sigma}=\frac{1}{2}$ is $\sigma \otimes \sigma$. Writing its OPE schematically,

\footnotetext{
${ }^{17}$ Varying the spatial dimension provides one indication that numerical errors have a large effect. Evaluating conformal blocks at $d=2.01$ instead of $d=2$ results in a much smaller bound on $\Delta_{*}$.
}

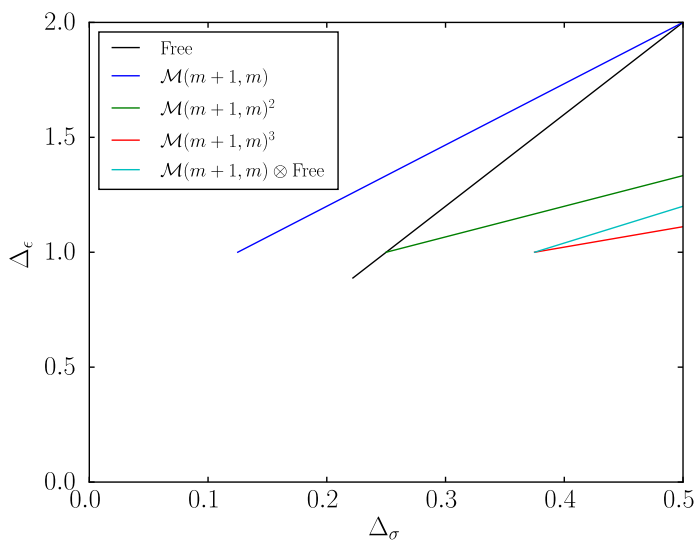

FIG. 6. Tensor product theories that are allowed in both sides of Fig. 1. Since we must have $\Delta_{\epsilon} \geq 1$, all other tensor products involving the free field vertex operators necessarily lie to the right.

$$
\begin{aligned}
(\sigma \otimes \sigma) \times(\sigma \otimes \sigma)= & (I \otimes I)+(I \otimes \epsilon)+(\epsilon \otimes I) \\
& +(\epsilon \otimes \epsilon)+\cdots
\end{aligned}
$$

includes two relevant operators. In order for these to have the same scaling dimension, the $\mathcal{M}(m+1, m) \otimes \mathcal{M}\left(m^{\prime}+\right.$ $\left.1, m^{\prime}\right)$ product must have $m=m^{\prime}$. Expanding the search to include free theories and generalized free theories, it is a simple exercise to check that the lines in Fig. 6 all have one relevant $\mathbb{Z}_{2}$-odd scaling dimension and one relevant $\mathbb{Z}_{2}$-even scaling dimension.

Evidently, it is not possible to isolate particular minimal models in a three-correlator bootstrap by specifying the number of relevant operators. One has to consider more stringent assumptions or add more correlators. The former approach was discussed already in [60], where it was found that the one-correlator Ising kink sharpens considerably when scalars are restricted to lie in $\mathcal{S}=\{\Delta>3\} \cup$ $\left\{\Delta=\Delta_{\epsilon}\right\}$. This kink becomes an island when a similar restriction is made for a three-correlator system. Following [61], it is likely that one can obtain this island from a single correlator by imposing large gaps in the spin- 0 and spin-2 sectors.

A more ambitious goal is to produce islands under minimal assumptions by introducing a third external scalar. Taking this external scalar to be odd, plots along the lines of Fig. 4 offer some preliminary insight. ${ }^{18}$ Regions where all $C_{\left(r_{1}, s_{1}\right)\left(r_{2}, s_{2}\right)}^{\left(r_{3}, s_{3}\right)}>0$ are likely to survive, but as Table VI shows, there can be several negative structure constants with more than three correlators. We have seen that the negative constant $C_{(1,3)(1,3)}^{(1,5)}$ in the three-correlator system was innocuous because it did not appear in any mixed

\footnotetext{
${ }^{18}$ The lightest $\mathbb{Z}_{2}$-odd scalar after $\sigma$ is $\sigma^{\prime} \equiv \phi_{1,4}$. The lightest $\mathbb{Z}_{2}$-even scalar after $\epsilon$ is $\epsilon^{\prime} \equiv\left[L_{-2} \bar{L}_{-2}, \phi_{1,1}\right]$. We have made the choice in which all operator fusions are between Virasoro primaries.
} 
TABLE VI. Virasoro block coefficients (other than the ones in Fig. 4) appearing in four-point functions made from $\sigma, \epsilon$, and $\sigma^{\prime}$. Only one is non-negative for all $m \geq 3$.

\begin{tabular}{ll}
\hline \hline Coefficients & \multicolumn{1}{c}{ Signs } \\
\hline$C_{(1,3)(1,4)}^{(1,3)}$ & Positive \\
$C_{(1,5)(1,4)}^{(1,4)}$ & Negative for $m<4$ \\
$C_{(1,4)(1,4)}^{(1,7)}$ & Negative for $m<6$ \\
$C_{(1,3)(1,4)}^{(1,6)}$ & Negative for $m<5$ \\
$C_{(1,2)(1,4)}^{(1,5)}$ & Negative for $m<4$ \\
\hline \hline
\end{tabular}

correlators. It is therefore encouraging that the coefficients $C_{(1,2)(1,4)}^{(1,5)}$ and $C_{(1,3)(1,4)}^{(1,6)}$, which have first-order zeros, participate in $\left\langle\sigma \sigma \sigma^{\prime} \sigma^{\prime}\right\rangle$ and $\left\langle\epsilon \epsilon \sigma^{\prime} \sigma^{\prime}\right\rangle$, respectively. Lest we become too encouraged, it is important to note that $\Delta_{\sigma^{\prime}}$ is defined as the starting point for a continuum of irrelevant operators. This represents a fundamental difference as compared to the one-correlator and three-correlator analysis. It remains to be seen whether we can still derive strong bounds from a scan over two isolated scaling dimensions and one nonisolated scaling dimension.

\section{B. Conformal manifolds}

Even though the tensor product lines above all have $\Delta_{\sigma}>\frac{1}{4}$, there could be other CFTs in $\frac{1}{8}<\Delta_{\sigma}<\frac{1}{5}$ with sufficiently few relevant operators to survive the constraints of $\sigma, \epsilon$, and $\sigma^{\prime}$. An interesting possibility that would complicate the search for islands is a continuous line of theories ending somewhere close to the Ising model. We may search for an example by using the extremal functional method on solutions to crossing that involve a scalar $\Phi$ of dimension $2 .{ }^{19}$ This provides another opportunity to predict a mixed correlator result without actually bootstrapping more than one correlator.

The first step is to maximize $\lambda_{\sigma \sigma \Phi}^{2}$ in the range $\frac{1}{8}<\Delta_{\sigma}<$ $\frac{1}{2}$ with the constraint that all scalar dimensions are above $2 \Delta_{\sigma}$. Each point saturating the bound yields a spectrum with the marginal deformation $\Phi$. To see that this set of solutions is not a conformal manifold, we may check that the central charge varies with $\Delta_{\sigma}$. Specifically, it reaches a minimum value of $c \approx 1.12$. It is then straightforward to force our putative theories to have this central charge (or any larger value) by taking

$$
F_{-, 0,0}^{\sigma \sigma ; \sigma \sigma}(u, v) \mapsto F_{-, 0,0}^{\sigma \sigma ; \sigma \sigma}(u, v)+\frac{\Delta_{\sigma}^{2}}{c} F_{-, 2,2}^{\sigma \sigma ; \sigma \sigma}(u, v)
$$

\footnotetext{
${ }^{19}$ The idea of building up a previously unknown conformal manifold was discussed in [62]. Their method uses large- $N$ perturbation theory to construct the holographic dual of a bulk action with shift-symmetric couplings. For a recent bootstrap study of a known conformal manifold, see [63].
}

in the usual one-correlator crossing equation. To prevent (4.4) from being undone by a second copy of the stress tensor, we have imposed the gap $\Delta_{T}>2.1$ on spin-2 operators. Performing a second pass in this way, we have found low-lying dimensions and OPE coefficients with examples plotted in Fig. 7. With constant $c$ and $\Delta_{\Phi}$, these CFT data appear to satisfy all constraints of a conformal manifold that are directly accessible to $\langle\sigma \sigma \sigma \sigma\rangle$. Despite this, there is a method from [29] that can be revived to gain indirect information about the next four-point function, which we will call $\langle\epsilon \epsilon \epsilon \epsilon\rangle$.

The key is that the dimensions in $\sigma \times \sigma$ are also the dimensions in any OPE between identical scalars. Given a sufficiently long list of scaling dimensions, there is no reason why a search for optimal OPE coefficients has to be done for $\sigma \times \sigma$ rather than $\epsilon \times \epsilon$. We confirm this in Appendix B, by taking an approximate Ising model spectrum and performing the same type of fit that was done in [29]. Our results show that the estimation of $\lambda_{\sigma \sigma \mathcal{O}}^{2}$ could have been extended to $\lambda_{\epsilon \epsilon \mathcal{O}}^{2}$ without the threecorrelator crossing equations. The fitting procedure therefore differs from the primal method of [45], which is more accurate but limited to the direct correlators under study. Assuming that the method can be trusted, at least for 2D theories, we now have access to $\epsilon \times \epsilon$ coefficients including $\lambda_{\epsilon \epsilon \Phi}$. This allows another property of conformal manifolds to be tested.

When CFTs are parametrized by an exactly marginal operator with coupling $g$, there is a complicated set of differential equations that their local data must satisfy $[64,65]$. The simplest of these is $\frac{\mathrm{d} \Delta_{i}}{\mathrm{~d} g}=-S_{d-1} \lambda_{i i \Phi}$ which reads $\frac{\mathrm{d} \Delta_{i}}{\mathrm{~d} g}=-2 \pi \lambda_{i i \Phi}$ in two dimensions. The constraint

$$
\frac{\mathrm{d} \Delta_{\epsilon}}{\mathrm{d} \Delta_{\sigma}}=\frac{\lambda_{\epsilon \epsilon \Phi}}{\lambda_{\sigma \sigma \Phi}}
$$

immediately follows. The two sides of this equation have been plotted in Fig. 8. Even though $\lambda_{\epsilon \epsilon \Phi}$ is not known with high precision, the noise in the data seems much too small to explain the violation of (4.5). We must conclude that if a conformal manifold in $\frac{1}{8}<\Delta_{\sigma}<\frac{1}{2}$ allowed by Fig. 1 exists, it is not privileged enough to be found by this onecorrelator exercise.

It would be very interesting to find other applications for the dual method in [29]. Unfortunately, Appendix B shows that results become much less reliable above $d=2$. A possible reason for this was given in [47] which noticed a surprising preference for double-twist operators in the three-dimensional (3D) numerical bootstrap. To review, double-twist families have the following schematic form for $\ell \rightarrow \infty$ : 


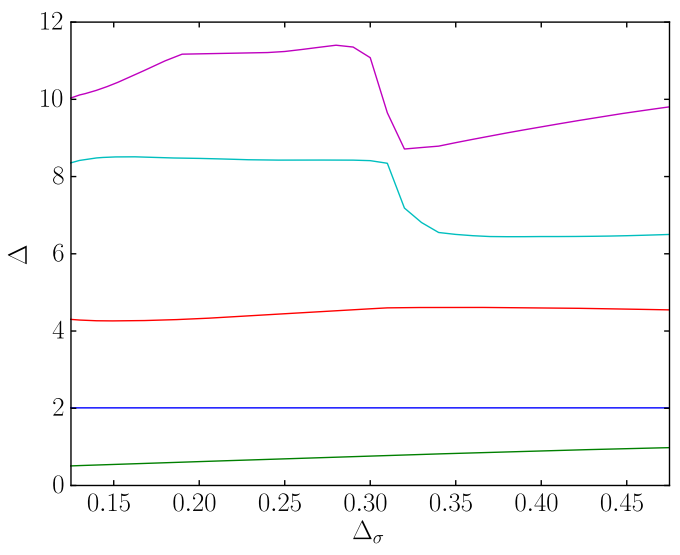

(a) Scaling dimensions

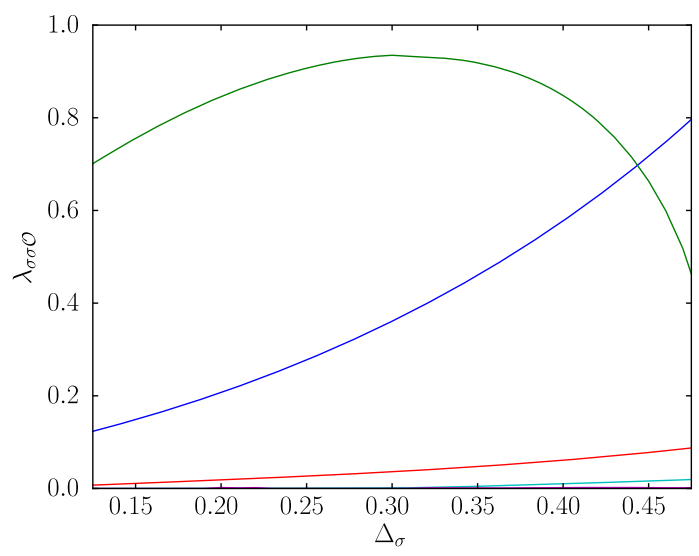

(b) OPE coefficients

FIG. 7. Dimensions and OPE coefficients for scalars in the spectrum maximizing $\lambda_{\sigma \sigma \Phi}^{2}$. The green and blue lines are $\epsilon$ and $\Phi$, respectively.(a) Spectrum as a function of $\Delta_{\sigma}$ which parametrizes the solution to crossing. (b) Three point couplings as a function of $\Delta_{\sigma}$ which parametrizes the solution to crossing.

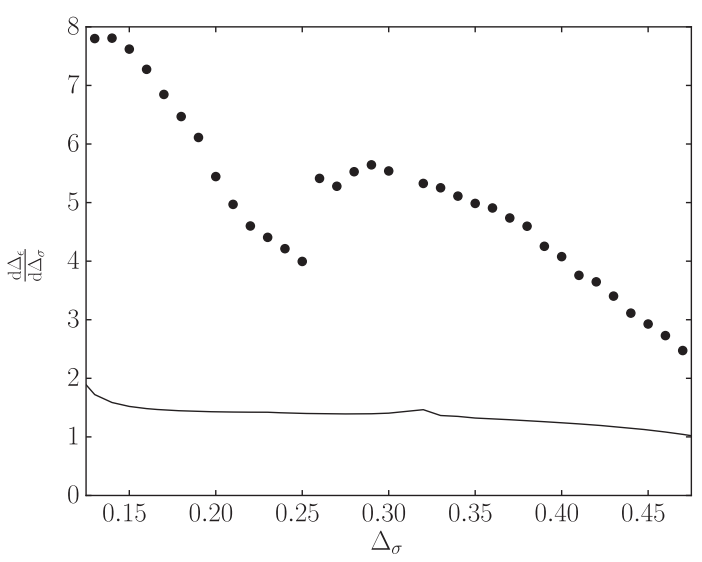

FIG. 8. The slope of $\Delta_{\epsilon}$ as one goes through the artificial spectra parametrized by $\Delta_{\sigma}$. The points, predicted by (4.5), would have to match if these operators were to come from a genuine theory with a dimensionless coupling. (a) Comparison for coefficients in the $\sigma \times \sigma$ OPE. (b) Comparison for coefficients in the $\varepsilon \times \varepsilon$ OPE.

$$
\begin{aligned}
{[\phi \phi]_{n} } & =\phi \partial^{\mu_{1}} \cdots \partial^{\mu_{\ell}} \square^{n} \phi, \\
\tau & =2 \tau_{\phi}+2 n+O\left(\ell^{-1}\right) .
\end{aligned}
$$

The light-cone bootstrap requires them to appear in any CFT with a twist gap [66,67]. When $d>2$, every operator except the identity has positive twist and the extremal functional method is able to find spectra dominated by (4.6). The absence of other operators interferes with our ability to fit OPE coefficients. Conversely, 2D theories have no need for double-twist operators as the identity, stress tensor, and higher-spin currents all have $\tau=0$. The quality of our fit suggests that this resolves the main source of bias in the extremal spectrum.

\section{Supersymmetric minimal models}

So far, we have been concerned with finding the necessary correlators to single out theories that are minimal with respect to the Virasoro algebra. However, the unitary representations of the $\mathcal{N}=1$ super-Virasoro algebra also admit a discrete series for central charges below that of the free field. In analogy with $\mathcal{M}(m+1, m)$, we can continue them to $\mathcal{S} \mathcal{M}(m+2, m)$ where noninteger $m$ breaks unitarity but preserves crossing symmetry. Studying these solved theories offers another route toward understanding the systematics of the bootstrap. It could also be interesting to compare results for the tricritical Ising model since this is the lowest model of $\mathcal{S M}(m+2, m)$ but the second lowest model of $\mathcal{M}(m+1, m)$.

The super-Virasoro graded commutation relations

$$
\begin{aligned}
{\left[L_{m}, L_{n}\right] } & =(m-n) L_{m+n}+\frac{c}{12} m(m-1)(m+1) \delta_{m+n, 0}, \\
{\left[L_{m}, G_{r}\right] } & =\left(\frac{m}{2}-r\right) G_{m+r} \\
\left\{G_{r}, G_{s}\right\} & =2 L_{r+s}+\frac{c}{3}\left(r-\frac{1}{2}\right)\left(r+\frac{1}{2}\right) \delta_{r+s, 0}
\end{aligned}
$$

actually describe two algebras since fermions do not have to be periodic in radial quantization. When the indices on $G_{r}$ are integers, Eq. (4.7) is the Ramond superalgebra; otherwise it is the Neveu-Schwarz superalgebra. The superVirasoro minimal models, which contain representations of each, have a Kac formula given by

$$
\begin{aligned}
& c= \frac{3}{2}-\frac{12}{m(m+2)} \quad m>2, \\
& h_{r, s}= \frac{[(m+2) r-m s]^{2}-4}{8 m(m+2)}+\frac{1}{32}\left[1-(-1)^{r-s}\right] \\
& r, s \in \mathbb{Z}_{>0} .
\end{aligned}
$$


If $r-s$ is even (odd), this is a Neveu-Schwarz (Ramond) degenerate weight [68]. In either case, it is degenerate at level $r s / 2$. It is clear by inspection that $G_{ \pm \frac{1}{2}}$ generate a subalgebra of (4.7) that is independent of $c$. For integer indices, on the other hand, no global subalgebra exists. A numerical bootstrap approach is therefore most readily accessible for the Neveu-Schwarz sectors of $\mathcal{N}=(1,1)$ theories.

In the global algebra, which is $\mathfrak{o} \mathfrak{a p}(2 \mid 1)$, primary operators may be written as superfields; $\Phi(z, \theta)=$ $\phi(z)+\theta \psi(z)$. The superspace distance, which enters in correlation functions, is $Z_{i j} \equiv z_{i}-z_{j}-\theta_{i} \theta_{j}$. Even though cross ratios in $\mathbb{R}^{d}$ all involve at least four points, invariant combinations in superspace may be built using three points as well. The quantity

$$
\eta=\frac{\theta_{1} Z_{23}+\theta_{2} Z_{31}+\theta_{3} Z_{12}+\theta_{1} \theta_{2} \theta_{3}}{\sqrt{Z_{12} Z_{23} Z_{31}}}
$$

is invariant under $\mathfrak{o} \mathfrak{g} \mathfrak{p}(2 \mid 1)$ [69]. As a result, the three-point function depends on more than just an OPE coefficient. The general expression for the chiral half is

$$
\begin{aligned}
& \left\langle\Phi_{1}\left(z_{1}, \theta_{1}\right) \Phi_{2}\left(z_{2}, \theta_{2}\right) \Phi_{3}\left(z_{3}, \theta_{3}\right)\right\rangle \\
& =\frac{\lambda_{123}(1+\zeta \eta)}{Z_{12}^{h_{1}+h_{2}-h_{3}} Z_{23}^{h_{2}+h_{3}-h_{1}} Z_{31}^{h_{3}+h_{1}-h_{2}}},
\end{aligned}
$$

where $\zeta$ is an arbitrary Grassman number. Until recently, such extra parameters were eliminated by restricting the superconformal bootstrap to correlators of BPS operators $[12,13]$. We may indeed impose shortening conditions on (4.10), but due to the small amount of supersymmetry, this would require us to give up a lot. To be annihilated by a supercharge, each external operator would have to be the identity in at least one of the $\mathfrak{o} \mathfrak{g} \mathfrak{p}(2 \mid 1)$ factors. It is therefore preferable to leave (4.10) in its most general form and use superconformal blocks that include unknown coefficients reflecting the presence of $\zeta$.

The authors of [54] computed some of the necessary blocks and introduced a framework that still allows the bootstrap to proceed. Their idea is to consider an entire multiplet at once, with the external correlators involving all combinations of a primary and its superdescendants. When this is carried out for $\mathcal{N}=(1,1)$, the allowed regions will have to include all points corresponding to the $\mathcal{S M}(m+2, m)$. The strongest statement we can make from this is that the line

$$
\Delta_{\epsilon}=\frac{8}{3} \Delta_{\sigma}
$$

must be inside the bound for $\Delta_{\sigma}<\frac{1}{8}$. This comes from choosing the Neveu-Schwarz fields $\sigma \equiv \phi_{2,2}$ and $\epsilon \equiv \phi_{3,3}$. Right at $\Delta_{\sigma}=\frac{1}{8}$, we find ourselves in the $c=1$ model where $\phi_{3,3}=\phi_{1,3}$ and the entire level- $\frac{3}{2}$ subspace decouples. To see this, we may check that

$$
|\chi\rangle=\left[G_{-\frac{3}{2}}-\frac{2}{2 h+1} L_{-1} G_{-\frac{1}{2}}\right]|h\rangle
$$

is the unique quasiprimary state. Computing the norm and setting $h \mapsto h_{3,3}$, we find

$$
\begin{aligned}
\langle\chi \mid \chi\rangle & =\frac{2\left(2 c h+c+6 h^{2}-9 h\right)}{3(2 h+1)} \\
& \mapsto \frac{(m-4)(m+6)}{m^{2}+2 m+8}
\end{aligned}
$$

with a first-order zero. This is the behavior that we saw for $m=3$ in the bosonic case, but now it occurs for $m=4$. The tricritical Ising model, which has no $\phi_{3,3}$ operator, lives at the point $\left(\Delta_{\sigma}, \Delta_{\epsilon}\right)=\left(\frac{1}{10}, \frac{1}{10}\right)$.

The above calculation shows that a global block coefficient in the supersymmetric generalized minimal model line becomes negative for $m<4$. If we are to see an associated kink, this line must saturate the bound on operator dimensions from the long multiplet bootstrap. This brings us to a crucial difference between $\mathcal{M}(m+$ $1, m)$ and the Neveu-Schwarz sector of $\mathcal{S M}(m+2, m)$. In the former case, we saw the correct saturation with (1.4). The same cannot hold for (4.11) because it is strictly below the line for vertex operators. Writing a CFT vertex operator as $e^{i q \phi(z)}$ and an Superconformal field theory vertex operator as $e^{i q \Phi(z, \theta)}$, the two important properties are $\Delta \propto$ $q^{2}$ and additivity of $q$. These lead to $\Delta_{\epsilon}=4 \Delta_{\sigma}$ which is allowed by the one-correlator region of Fig. 1. Due to the restriction on the number of relevant operators, the threecorrelator region omits this line until $\Delta_{\sigma}=\frac{2}{9}$. It is therefore clear that treating four copies of the same multiplet with the methods of [54] is not enough. If our goal is to see a minimal model kink, the $\mathcal{N}=(1,1)$ bootstrap will require multiple correlators at the superspace level. Since each of these must separately expand to a mixed correlator system, the resulting problem is likely to be numerically intensive.

\section{CONCLUSION}

Through a combination of analytic techniques and onecorrelator numerics, we have explained an important aspect of Fig. 1-we have shown that the constraints on four-point functions of relevant operators are not strong enough to exclude the generalized minimal models. Constraints that are strong enough may be used in the conformal or superconformal bootstrap, but only when the number of four-point functions exceeds the maximum system size that has been tested to date.

Our analysis proceeded correlator by correlator. For $\langle\sigma \sigma \sigma \sigma\rangle$, the results were exact. The expressions (3.17) and (3.14) ensured that the global block coefficients 
involved were all positive. For $\langle\sigma \sigma \epsilon \epsilon\rangle$, all but one coefficient appeared to be positive upon using the approach of [17] — we simply expanded in $\mathfrak{g l}(2)$ blocks to high order and conjectured that the pattern continues to hold. For $\langle\epsilon \epsilon \epsilon \epsilon\rangle$, two pieces of the $\mathcal{M}(m+1, m)$ line had to be treated separately. In $4<m<\infty$, it was enough to compute coefficients again and see that there were no obvious signs of unitarity violation. However, $3<m<4$ required an exotic solution having only partial overlap with the CFT data of a generalized minimal model. It was possible to see evidence of this in the one-correlator bootstrap because of a special property of $\phi_{1,5}$ operators, namely their absence from the superselection sector $\sigma \times \sigma$. This had interesting implications for the uniqueness results in [46].

A possible future endeavor is to put all correlators on the same footing as $\langle\sigma \sigma \sigma \sigma\rangle$. In order to do this, one does not necessarily have to solve for $c_{2 n}^{\epsilon \epsilon(1, s) \epsilon \epsilon}$ as a known special function. The $c_{2 n}^{\sigma \sigma(1, s) \sigma \sigma}$ were ultimately shown to be positive using only the recursion for Wilson polynomials. Instead, the main challenge in extending the positivity proof is expressing global block coefficients as solutions of one recurrence relation instead of two. Our current approach, based on the BPZ equation, is awkward in this respect. It uses the Frobenius method to evaluate Taylor coefficients and then feeds these into a second recursion to obtain global block coefficients. It is worth checking if hypergeometric identities can be used to derive a recursion that operates on global block coefficients directly. It would also be interesting to take a closer look at super-BPZ equations. Our discussion surrounding the vanishing norm (4.13) can be made more systematic if we also check how other $\mathfrak{o} \mathfrak{g} \mathfrak{p}(2 \mid 1)$ blocks appear. BPZ differential equations in superspace have been studied in $[68,69]$ and some of them are second-order. This is exactly what we need to go beyond recurrence relations and apply the decompositions in $[21,22]$. Additionally, these methods could be applicable to the Knizhnik-Zamolodchikov equations associated with extended chiral algebras. A realistic hope is using them to explain a numerical bound in [70] which interpolates between $\mathcal{W}_{3}$-minimal models.

The last possibility we have discussed is an extension of the extremal functional method-reviving the fit in [29] to estimate more OPE coefficients than the ones that are known to high precision. While we only used this to demonstrate a null result, it would be interesting to find a further use for it in two dimensions. Complications in higher dimensions arise due to the privileged role of double-twist operators in the numerical bootstrap, a result that is not fully understood [47]. One should be able to get a sense of how robust it is by studying alternative bootstrap algorithms such as the one in [71].

\section{ACKNOWLEDGMENTS}

This work was partially supported by the Natural Sciences and Engineering Research Council of Canada.
Numerical results in this paper were obtained using the high-performance computing system at the Institute for Advanced Computational Science at Stony Brook University. I am grateful for discussions with Shai Chester, Liam Fitzpatrick, Anton de la Fuente, Matthijs Hogvervorst, Jaehoon Lee, Madalena Lemos, Dalimil Mazáč, Miguel Paulos, Leonardo Rastelli, Slava Rychkov, David Simmons-Duffin, Balt van Rees, and Alessandro Vichi. Most of these took place at various meetings of the Simons Collaboration on the Nonperturbative Bootstrap.

\section{APPENDIX A: LINEAR DIFFERENCE EQUATIONS}

We have regularly encountered linear recursions with three terms such as (2.13) and (3.17). Asymptotic analysis of sequences obeying these relations is a well understood subject, going by the name Birkhoff-Trjitzinsky theory. The following theorem summarizes a number of results from it [72].

Theorem 1. Let $y_{1}(n)$ and $y_{2}(n)$ be the two linearly independent solutions of the difference equation

$$
y(n+2)+a(n) y(n+1)+b(n) y(n)=0,
$$

where the coefficients have asymptotic expansions $a(n) \sim$ $\sum_{s=0}^{\infty} \frac{a_{s}}{n^{s}}$ and $b(n) \sim \sum_{s=0}^{\infty} \frac{b_{s}}{n^{s}}$.

(1) If the characteristic equation $\rho^{2}+a_{0} \rho+b_{0}=0$ has two distinct roots $\rho_{1}$ and $\rho_{2}$, the solutions satisfy $y_{j}(n) \sim \rho_{j}^{n} n^{\alpha_{j}} \sum_{s=0}^{\infty} \frac{c_{s, j}}{n^{s}}$ where $\alpha_{j}=\frac{a_{1} \rho_{j}+b_{1}}{a_{0} \rho_{j}+2 b_{0}}$.

(2) Otherwise, consider the double root $\rho$. If the auxiliary equation $a_{1} \rho+b_{1}=0$ is not satisfied, the solutions satisfy $y_{j}(n) \sim \rho^{n} e^{(-1)^{j} \beta \sqrt{n}} n^{\alpha} \sum_{s=0}^{\infty}(-1)^{j s} \frac{c_{s}}{n^{s / 2}}$ where $\alpha=\frac{1}{4}+\frac{b_{1}}{2 b_{0}}$ and $\beta=2 \sqrt{\frac{a_{0} a_{1}-2 b_{1}}{2 b_{0}}}$.

(3) Otherwise, consider the roots $\alpha_{1}$ and $\alpha_{2}$ of the indicial equation $\alpha(\alpha-1) \rho^{2}+\left(a_{1} \alpha+a_{2}\right) \rho+b_{2}=0$, ordered according to $\Re \alpha_{2} \geq \Re \alpha_{1}$. If $\alpha_{2}-\alpha_{1} \notin \mathbb{Z}_{\geq 0}$, the solutions satisfy $y_{j}(n) \sim \rho^{n} n^{\alpha_{j}} \sum_{s=0}^{\infty} \frac{c_{s, j}}{n^{s}}$.

(4) Otherwise, let $m=\alpha_{2}-\alpha_{1}$. The asymptotic expansion for the first solution is unchanged from the previous case but for the second solution we must use $y_{2}(n) \sim \rho^{n} n^{\alpha_{2}}\left(\sum_{s=0}^{\infty} \frac{d_{s}}{n^{s}}-\frac{d_{m}}{n^{m}}\right)+c \log (n) y_{1}(n)$.

Taking (A1) to be the recurrence relation for Wilson polynomials, we find the roots $\rho=1, \alpha_{1}=-2(a+x)$, and $\alpha_{2}=-2(a-x)$ in the third case of the theorem. Each coefficient in (3.16) can then be written as a linear combination of two functions asymptotic to power laws. As seen in Table VII, all of them decay to zero. When comparing to the results of [73,74], one must remember that $c_{2 n}^{\sigma \sigma(1,1) \sigma \sigma}$ and $c_{2 n}^{\sigma \sigma(1,3) \sigma \sigma}$ include many squared OPE coefficients due to the increasing amount of degeneracy at each level of a Verma module. 
TABLE VII. Decay rates of the fundamental solutions that comprise two of our main results. We have not included the prefactors in (3.16). These will make the convergence much faster, namely $(1 / 16)^{n}$, which can be predicted from the growth rate of $K_{2 n}(1)$.

\begin{tabular}{ll}
\hline \hline Coefficient & \multicolumn{1}{c}{ Leading rates } \\
\hline$c_{2 n}^{\sigma \sigma(1,1) \sigma \sigma}$ & $n^{-\frac{8+2 \Delta_{\sigma}}{3}}$ and $n^{2 \Delta_{\sigma}-2}$ \\
$c_{2 n}^{\sigma \sigma(1,3) \sigma \sigma}$ & $n^{-\frac{5+8 \Delta_{\sigma}}{3}}$ and $n^{-1}$ \\
\hline \hline
\end{tabular}

Our main claim about $c_{2 n}^{\sigma \sigma(1,1) \sigma \sigma}$ and $c_{2 n}^{\sigma \sigma(1,3) \sigma \sigma}$ — that they are positive for finite $n$-cannot be proven with asymptotics. Instead, we will use a theorem from [75] which bounds the ratio between neighboring terms in a sequence.

Theorem 2. Let $x(n)$ be a solution of

$$
x(n) \geq \frac{a(n)}{b(n)} x(n-1)-\frac{c(n)}{d(n)} x(n-2),
$$

where $a(n), b(n), c(n)$, and $d(n)$ are degree- $k$ polynomials with positive leading terms. Also define $f(n)=a(n+$ 1) $d(n+1)-\frac{2 b_{k}}{a_{k}} b(n+1) c(n+1)-\frac{a_{k}}{2 b_{k}} b(n+1) d(n+1)$. Finally, let $m$ be an integer large enough to guarantee that $b(n), c(n), d(n)$, and $f(n)$ have positive values for $n \geq m$. If $\frac{x(m)}{x(m-1)}>\frac{a_{k}}{2 b_{k}}$, then $\frac{x(n)}{x(n-1)}>\frac{a_{k}}{2 b_{k}}$ for $n \geq m$.

Proposition 1. The sequences $y_{1}(n)=P_{n}\left(\frac{7-2 \Delta_{\sigma}}{6}, \frac{4-2 \Delta_{\sigma}}{6}\right.$, $\left.-\frac{1-2 \Delta_{\sigma}}{6}, \frac{5+2 \Delta_{\sigma}}{6} ; \frac{1+4 \Delta_{\sigma}}{6}\right)$ and $y_{2}(n)=P_{n}\left(\frac{2+2 \Delta_{\sigma}}{3}, \frac{1}{2},-\frac{1}{2},-\frac{1}{2}\right.$; $\frac{1+4 \Delta_{\sigma}}{6}$ ) of Wilson polynomials are positive for $\frac{1}{8} \leq \Delta_{\sigma} \leq \frac{1}{2}$.

Proof.-The theorem above is more effective at identifying increasing sequences than ruling out changes of sign directly. Therefore, we will work with $x_{1}(n)=n^{2} y_{1}(n)$ and $x_{2}(n)=n^{2} y_{2}(n)$ - expressions that have been guided by the asymptotics in Table VII.

Looking at the more difficult case first, $x_{1}(n)$ satisfies (A2) with

$$
\begin{aligned}
& b(n)=(n-1)^{2}(n+1)(2 n+1)(4 n-3)\left(6 n-4 \Delta_{\sigma}+5\right), \\
& c(n)=n(n-1)(2 n-1)(2 n-3)(4 n+1)\left(3 n+2 \Delta_{\sigma}-4\right), \\
& d(n)=(n-2)^{2}(n+1)(2 n+1)(4 n-3)\left(6 n-4 \Delta_{\sigma}+5\right) .
\end{aligned}
$$

These are clearly positive for $n>2$. Also, by writing out the polynomial for $a(n)$, we find a leading coefficient of $a_{6}=2 b_{6}=96$. It remains to check $f(n)$ or equivalently $(n+2)^{-1}(2 n+3)^{-1}(4 n+1)^{-1}\left(6 n-4 \Delta_{\sigma}+11\right)^{-1} f(n)$. This is a sixth degree polynomial in which $64 \Delta_{\sigma}\left(\Delta_{\sigma}+\right.$ 1) $n^{6}$ is followed immediately by negative coefficients. From this we see that the critical value of $n$, beyond which $f(n)>0$, increases without bound as $\Delta_{\sigma} \rightarrow 0$. This reflects the fact that the $n^{2}$ we introduced is only able to overpower $n^{2 \Delta_{\sigma}-2}$ for strictly positive $\Delta_{\sigma}$. Fortunately, the smallest value of $\Delta_{\sigma}$ that we consider is $\frac{1}{8}$ leading to a critical value of $n=44$. Since $x_{1}(44)>x_{1}(43)$, we establish positivity of the entire sequence $x_{1}(n)$ by checking its first 44 terms.

Things will be easier for $x_{2}(n)$ which satisfies (A2) for

$$
\begin{aligned}
b(n)= & (n-1)^{2}\left(6 n+4 \Delta_{\sigma}-11\right)\left(6 n+4 \Delta_{\sigma}-5\right)^{2} \\
& \times\left(6 n+4 \Delta_{\sigma}+1\right)\left(12 n+4 \Delta_{\sigma}-23\right), \\
c(n)= & 1296 n^{2}(n-1)(n-3)\left(12 n+4 \Delta_{\sigma}-11\right), \\
d(n)= & \left(6 n+4 \Delta_{\sigma}-11\right)\left(6 n+4 \Delta_{\sigma}-5\right)^{2} \\
& \times\left(6 n+4 \Delta_{\sigma}+1\right)\left(12 n+4 \Delta_{\sigma}-23\right) .
\end{aligned}
$$

Additionally, we find $a_{6}=2 b_{6}=31104$ and an $f(n)$ proportional to $\left(6 n+4 \Delta_{\sigma}-5\right)\left(6 n+4 \Delta_{\sigma}+1\right)^{2}(6 n+$ $\left.4 \Delta_{\sigma}+7\right)\left(12 n+4 \Delta_{\sigma}-11\right)$. The nontrivial factor of $f(n)$ begins with $41472\left(\Delta_{\sigma}-\frac{1}{8}\right) n^{5}$ which is potentially problematic, but the next coefficient that follows it is positive for all real $\Delta_{\sigma}$. As a result, $f(n)$ is positive for $n>3$ just as the polynomials above. Checking that $x_{2}(3)>$ $x_{2}(2)>x_{2}(1)>0$, positivity of $x_{2}(n)$ has been proven as well.

Although we do not have closed-form solutions for them, it is possible that $c_{2 n}^{\epsilon \epsilon(1,1) \epsilon \epsilon}, c_{2 n}^{\epsilon \epsilon(1,3) \epsilon \epsilon}$, and $c_{2 n}^{\epsilon \epsilon(1,5) \epsilon \epsilon}$ are positive as well. The main hint of this, which we now prove, is that the Virasoro blocks containing them have positive Taylor coefficients around $z=0$. The fact that this is a necessary condition follows trivially from expanding $g(z)=\sum_{n=0}^{\infty} c_{2 n} K_{r+2 n}(z)$. The analogous statement in higher dimensions was proven in [76].

Proposition 2. Let $b_{k}$ be a sequence starting at $b_{0}=1$ with the rest of the terms given by (3.21). If $\Delta_{\epsilon}>1$, the sequence monotonically increases.

Proof.-Defining $K=k+r$ for brevity, we have

$$
\begin{aligned}
& {\left[4 K^{3}+8\left(1-\Delta_{\epsilon}\right) K^{2}+\left(3 \Delta_{\epsilon}^{2}-14 \Delta_{\epsilon}+4\right) K+3 \Delta_{\epsilon}\left(\Delta_{\epsilon}-2\right)\right] b_{k+1}=\left[12 K^{2}-4\left(5 \Delta_{\epsilon}+1\right) K+6 \Delta_{\epsilon}^{2}\right] K b_{k}} \\
& \quad-\left[12 K^{3}-16\left(\Delta_{\epsilon}+2\right) K^{2}+2\left(\Delta_{\epsilon}^{2}+12 \Delta_{\epsilon}+14\right) K+2\left(\Delta_{\epsilon}-2\right)\left(\Delta_{\epsilon}+1\right)\left(\Delta_{\epsilon}+2\right)\right] b_{k-1} \\
& \quad+\left[4 K^{3}-4\left(\Delta_{\epsilon}+5\right) K^{2}-\left(\Delta_{\epsilon}^{2}-10 \Delta_{\epsilon}-32\right) K+\left(\Delta_{\epsilon}-2\right)\left(\Delta_{\epsilon}+2\right)\left(\Delta_{\epsilon}+4\right)\right] b_{k-2} .
\end{aligned}
$$

Although this has four terms, we will convert it to a simpler recursion having only three. We do this by assuming that the piece with $b_{k-1}$ and $b_{k-2}$ is bounded by some function of $b_{k}$ and $b_{k-1}$. Our ansatz for this function is $\left[4 K^{2}-12\left(\Delta_{\epsilon}+1\right) K+M\right] K b_{k}+4 K^{3} b_{k-1}$. In other words, we need to show that 


$$
\begin{aligned}
& {\left[4 K^{2}-12\left(\Delta_{\epsilon}+1\right) K+M\right] K b_{k}>\left[8 K^{3}-16\left(\Delta_{\epsilon}+2\right) K^{2}\right.} \\
& \left.\quad+2\left(\Delta_{\epsilon}^{2}+12 \Delta_{\epsilon}+14\right) K+2\left(\Delta_{\epsilon}-2\right)\left(\Delta_{\epsilon}+1\right)\left(\Delta_{\epsilon}+2\right)\right] b_{k-1} \\
& \quad-\left[4 K^{3}-4\left(\Delta_{\epsilon}+5\right) K^{2}-\left(\Delta_{\epsilon}^{2}-10 \Delta_{\epsilon}-32\right) K\right. \\
& \left.\quad+\left(\Delta_{\epsilon}-2\right)\left(\Delta_{\epsilon}+2\right)\left(\Delta_{\epsilon}+4\right)\right] b_{k-2} .
\end{aligned}
$$

Using (A6) in (A5), we find an expression of the form $R_{+}(K) b_{k+1}>R_{0}(K) b_{k}+R_{-}(K) b_{k-1}$. We will perform a rescaling to instead write this as

$$
\begin{aligned}
S_{+}(K) b_{k+1}> & S_{0}(K) b_{k}+S_{-}(K) b_{k-1} \\
S_{i}(K) \equiv & {\left[4(K+1)^{2}-12\left(\Delta_{\epsilon}+1\right)(K+1)+M\right] } \\
& \times(K+1) R_{+}(K)^{-1} R_{i}(K) .
\end{aligned}
$$

For our assumption to be true, the fractional coefficients in (A7) must exceed the $K \mapsto K+1$ versions of the ones in (A6). These two conditions each give one side of an inequality for $M$. The result is $10 \Delta_{\epsilon}^{2}+20 \Delta_{\epsilon}+4<M<$ $10 \Delta_{\epsilon}^{2}+29 \Delta_{\epsilon}+4$ which may be satisfied for any $\Delta_{\epsilon}>0$. Having chosen $M$ appropriately, we have moved the problem into the domain of the theorem above. The monotonicity proof for $b_{k}$ is now identical to the one for $n^{2} c_{2 n}^{\sigma \sigma(1,1) \sigma \sigma}$ and $n^{2} c_{2 n}^{\sigma \sigma(1,3) \sigma \sigma}$.

\section{APPENDIX B: IMPLEMENTATION DETAILS}

\section{The semidefinite program}

The conformal bootstrap is any technique for demanding that crossing symmetry and unitarity hold for the four-point function:

$$
\begin{aligned}
& \left\langle\phi_{i}\left(x_{1}\right) \phi_{j}\left(x_{2}\right) \phi_{k}\left(x_{3}\right) \phi_{l}\left(x_{4}\right)\right\rangle \\
& =\left(\frac{\left|x_{24}\right|}{\left|x_{14}\right|}\right)^{\Delta_{i j}}\left(\frac{\left|x_{14}\right|}{\left|x_{13}\right|}\right)^{\Delta_{k l}} \frac{\sum_{\mathcal{O}} \lambda_{i j \mathcal{O}} \lambda_{k l \mathcal{O}} g_{\mathcal{O}}^{\Delta_{i j}, \Delta_{k l}}(u, v)}{\left|x_{12}\right|^{\Delta_{i}+\Delta_{j}}\left|x_{34}\right|^{\Delta_{k}+\Delta_{l}}} .
\end{aligned}
$$

The conformal blocks $g_{\mathcal{O}}^{\Delta_{i j}, \Delta_{k l}}(u, v)$ are functions of the cross ratios $u=\frac{x_{12}^{2} x_{34}^{2}}{x_{13}^{2} x_{24}^{2}}$ and $v=\frac{x_{14}^{2} x_{23}^{2}}{x_{13}^{2} x_{24}^{2}}$. Invariance under $(1, i) \leftrightarrow(3, k)$, which relates two channels of crossing symmetry, leads to the following sum rule [14]:

$$
\begin{aligned}
& \sum_{\mathcal{O}}\left[\lambda_{i j \mathcal{O}} \lambda_{k l \mathcal{O}} F_{\mp, \mathcal{O}}^{i j ; k l}(u, v) \pm \lambda_{k j \mathcal{O}} \lambda_{i l \mathcal{O}} F_{\mp, \mathcal{O}}^{k j ; i l}(u, v)\right]=0, \\
& F_{ \pm, \mathcal{O}}^{i j ; k l} \equiv v^{\frac{\Delta_{k}+\Delta_{j}}{2}} g_{\mathcal{O}}^{\Delta_{i j}, \Delta_{k l}}(u, v) \pm u^{\frac{\Delta_{k}+\Delta_{j}}{2}} g_{\mathcal{O}}^{\Delta_{i j}, \Delta_{k l}}(v, u) .
\end{aligned}
$$

To apply this rule, we choose an odd scalar $\sigma$ and an even scalar $\epsilon$ and let our external operators run over all admissible combinations of these. Using $\lambda_{\sigma \epsilon \mathcal{O}}=(-1)^{\ell} \lambda_{\epsilon \sigma \mathcal{O}}$, this yields

$$
\sum_{\mathcal{O}, 2 \mid \ell}\left(\lambda_{\sigma \sigma \mathcal{O}} \lambda_{\epsilon \epsilon \mathcal{O}}\right) V_{+, \Delta, \ell}\left(\begin{array}{c}
\lambda_{\sigma \sigma \mathcal{O}} \\
\lambda_{\epsilon \epsilon \mathcal{O}}
\end{array}\right)+\sum_{\mathcal{O}} \lambda_{\sigma \epsilon \mathcal{O}}^{2} V_{-, \Delta, \ell}=0
$$

where

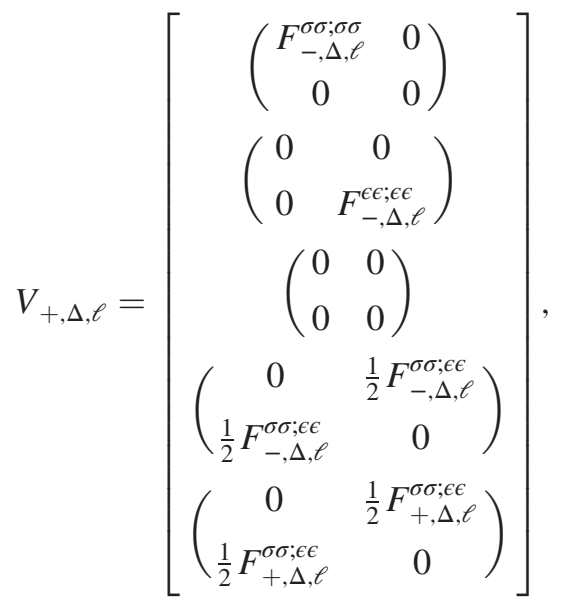

$$
\begin{aligned}
& V_{-, \Delta, \ell}=\left[\begin{array}{c}
0 \\
0 \\
F_{-, \Delta, \ell}^{\sigma \epsilon ; \sigma \epsilon} \\
(-1)^{\ell} F_{-, \Delta, \ell}^{\epsilon \sigma ; \sigma \epsilon} \\
-(-1)^{\ell} F_{+, \Delta, \ell}^{\epsilon \sigma \sigma \epsilon}
\end{array}\right] .
\end{aligned}
$$

In ruling out solutions to (B3), which is a set of five functional equations, we must approximate each row as a finite-dimensional vector. The standard way to do this is to expand around the point $(u, v)=\left(\frac{1}{4}, \frac{1}{4}\right)$. We may take either derivatives with respect to $z, \bar{z}$, defined by $u=|z|^{2}$, $v=|1-z|^{2}$, or the diagonal/off-diagonal variables $a=$ $z+\bar{z}, b=(z-\bar{z})^{2}$ [29]. We choose $a, b$ and control the order of our derivatives $\frac{\partial^{m+n} g_{\Delta, \ell}}{\partial a^{m} \partial b^{n}}$ with two parameters $m_{\max }$ and $n_{\max }$ :

$$
\begin{aligned}
& n \in\left\{0, \ldots, n_{\max }\right\} \\
& m \in\left\{0, \ldots, 2\left(n_{\max }-n\right)+m_{\max }\right\}
\end{aligned}
$$

Since half of the derivatives vanish when our conformal blocks are added or subtracted, the resulting number of components is

$$
N=\left\lfloor\left(n_{\max }+1\right)\left(m_{\max }+n_{\max }+1\right) / 2\right\rfloor .
$$

The three-correlator plot in this work was obtained with $\left(m_{\max }, n_{\max }\right)=(3,5)$. All one-correlator results, on the other hand, use $\left(m_{\max }, n_{\max }\right)=(5,10)$. There are two additional parameters needed to turn (B3) into a concrete bootstrapping problem. One is a cutoff on the number of spins, which we take to be $\ell_{\max }=30$. The other is the accuracy parameter for a single conformal block, which we take to be $k_{\max }=40$. This controls how many poles from the triple series 


$$
\begin{aligned}
& \Delta_{1}(\ell)=1-\ell-k \quad k=1,2, \ldots, \\
& \Delta_{2}(\ell)=\frac{d}{2}-k \quad k=1,2, \ldots, \\
& \Delta_{3}(\ell)=d-1+\ell-k \quad k=1,2, \ldots, \ell
\end{aligned}
$$

appear in the function

$$
\begin{aligned}
\chi_{\ell}(\Delta) & =\frac{r_{*}^{\Delta}}{\Pi_{i}\left(\Delta-\Delta_{i}(\ell)\right)}, \\
r_{*} & \equiv 3-2 \sqrt{2} .
\end{aligned}
$$

As explained in $[14,77,78]$, there are algorithms for explicitly constructing each conformal block derivative as a rational approximation:

$$
\frac{\partial^{m+n}}{\partial a^{m} \partial b^{n}} F_{ \pm, \Delta, \ell}^{i j ; k l}(a=1, b=0)=\chi_{\ell}(\Delta) P_{ \pm, \ell}^{i j ; k l ; m n}(\Delta) .
$$

Here, $P_{ \pm, \ell}^{i j ; k l ; m n}$ is a polynomial with the same degree as $\chi_{\ell}$ for $m=n=0$. Its degree goes up by one whenever the derivative order is increased. Our task of inputting $\left(k_{\max }, \ell_{\max }, m_{\max }, n_{\max }\right)$ and computing a table suitable for approximating (B3) is accomplished with the program PyCFTBOOT [79].

With the truncations described above, problems of this form are tractable with semidefinite programming [15]. In the dual formulation, one wishes to find a linear functional $\mathbf{y}$ which sends each term of (B3) to a positive-definite matrix, thereby certifying that no solution to crossing symmetry exists. For illustrative purposes, we consider a single correlator problem which allows us to drop the $i j ; \mathrm{kl}$ and \pm labels on $P_{ \pm, \ell}^{i j ; k l ; m n}$. We will also drop $m n$ through our understanding that $\mathbf{P}_{\ell}$ is a vector with components $P_{\ell}^{i}$. If we single out the contribution of the identity operator as $\mathbf{n}$, we arrive at the polynomial matrix program (PMP) where we include an objective b for generality,

$\operatorname{maximize} \mathbf{b}^{\mathrm{T}} \mathbf{y}$ over $\mathbf{n}^{\mathrm{T}} \mathbf{y}=1$,

such that $\mathbf{P}_{\ell}(\Delta)^{\mathrm{T}} \mathbf{y} \geq 0$ for all $\ell \leq \ell_{\max }, \Delta \geq \Delta_{\text {min }}$.

After all, the crossing equation

$$
\sum_{k, \ell} \lambda_{k, \ell}^{2} \mathbf{P}_{\ell}\left(\Delta_{k}\right)=\mathbf{n}
$$

becomes a contradiction when $\mathbf{y}$ solving the above conditions is applied to both sides. If we reshuffle each vector according to

$$
\begin{aligned}
& \tilde{P}_{\ell}^{0}=\frac{1}{n^{0}} P_{\ell}^{0}, \\
& \tilde{P}_{\ell}^{i}=P_{\ell}^{i}-\frac{n^{i}}{n^{0}} P_{\ell}^{0},
\end{aligned}
$$

dotting $\mathbf{P}_{\ell}$ with a functional whose action on $\mathbf{n}$ is 1 becomes the same as dotting $\tilde{\mathbf{P}}_{\ell}$ with a functional whose leading component is 1 . This is precisely the choice to work with crossing equations projectively as (B11) becomes

$$
\sum_{k, \ell} \lambda_{k, \ell}^{2}\left[\begin{array}{l}
\tilde{P}_{\ell}^{0}\left(\Delta_{k}\right) \\
\tilde{P}_{\ell}^{i}\left(\Delta_{k}\right)
\end{array}\right]=\left[\begin{array}{l}
1 \\
0
\end{array}\right]
$$

after reshuffling both sides. Any spectrum satisfying the bottom row can automatically be made to satisfy the top row through a rescaling. From now on, we will denote the polynomial vector in the bottom row of (B13) by $\mathbf{P}_{\ell}$ to rewrite the PMP,

$\operatorname{maximize} \mathbf{b}^{\mathrm{T}} \mathbf{y}$

such that $P_{\ell}^{0}(x)+\mathbf{P}_{\ell}(x)^{\mathrm{T}} \mathbf{y} \geq 0$ for all $\ell \leq \ell_{\max }, x \geq 0$.

Here, $x=\Delta-\Delta_{\min }$. To solve this type of problem efficiently, we use the program SDPB [15]. Because we will see an alternative choice shortly, we briefly review the process by which SDPB translates (B14) into a semidefinite program (SDP).

Positivity of $P_{\ell}^{0}(x)+\mathbf{P}_{\ell}(x)^{\mathrm{T}} \mathbf{y}$ on the half-line is equivalent to the requirement that it be equal to

$$
\operatorname{Tr}\left(\left[\begin{array}{cc}
\mathbf{q}_{\ell}(x) \mathbf{q}_{\ell}^{\mathrm{T}}(x) & 0 \\
0 & x \mathbf{q}_{\ell}(x) \mathbf{q}_{\ell}^{\mathrm{T}}(x)
\end{array}\right] Y_{\ell}\right),
$$

where $Y_{\ell}$ is positive definite and $\mathbf{q}_{\ell}$ is a vector of orthogonal polynomials. We have abused notation slightly since the maximum power of $x$ that (B15) needs to express may be even or odd. Because of this, the first $\mathbf{q}_{\ell}$ might have one more component than the second $\mathbf{q}_{\ell}$. It is sufficient to demand this equality on a set of sample points which we denote $x_{k}$. It is also possible to combine all $Y_{(k, \ell)}$ into a single matrix $Y$. Making the identifications

$$
\begin{aligned}
A_{(k, \ell)}= & \operatorname{diag}\left(0, \ldots, 0,\left[\begin{array}{cc}
\mathbf{q}_{\ell}\left(x_{k}\right) \mathbf{q}_{\ell}^{\mathrm{T}}\left(x_{k}\right) & 0 \\
0 & x_{k} \mathbf{q}_{\ell}\left(x_{k}\right) \mathbf{q}_{\ell}^{\mathrm{T}}\left(x_{k}\right)
\end{array}\right],\right. \\
& 0, \ldots, 0), \\
B_{(k, \ell), i}= & -P_{\ell}^{i}\left(x_{k}\right), \\
c_{(k, \ell)}= & P_{\ell}^{0}\left(x_{k}\right), \\
C & =0,
\end{aligned}
$$

(B14) becomes

$$
\begin{aligned}
& \text { maximize } \operatorname{Tr}(C Y)+\mathbf{b}^{\mathrm{T}} \mathbf{y} \text { over } Y \succeq 0, \\
& \text { such that } \operatorname{Tr}\left(A_{*} Y\right)+B \mathbf{y}=\mathbf{c} .
\end{aligned}
$$


In the numerical bootstrap, (B17) and the primal problem corresponding to it are typically solved together, in order to see which one becomes feasible first [15].

\section{The extremal functional method}

It was shown in [29] that solutions to crossing symmetry may be built by locating the zeros of $\mathbf{y}$. This functional may be found either by ruling out CFTs just outside the allowed region or by maximizing an OPE coefficient just inside it. Ideally, elements of the spin- $\ell$ spectrum are dimensions $\Delta_{k}$ such that

$\operatorname{det}\left(\mathbf{y}^{\mathrm{T}}\left[\begin{array}{ccc}\left(\mathbf{P}_{\ell}\left(\Delta_{k}\right)\right)_{0,0} & \cdots & \left(\mathbf{P}_{\ell}\left(\Delta_{k}\right)\right)_{0, n} \\ \vdots & \ddots & \vdots \\ \left(\mathbf{P}_{\ell}\left(\Delta_{k}\right)\right)_{n, 0} & \cdots & \left(\mathbf{P}_{\ell}\left(\Delta_{k}\right)\right)_{n, n}\end{array}\right]\right)=0$

where $\mathbf{P}_{\ell}$ is one of the polynomial vectors appearing in (B9). On the other hand, numerical errors usually prevent this polynomial from ever reaching zero for real $\Delta$. There are two approaches, both based on the SPECTRUM.PY script [47], which make it easy to account for this. The first is to run the script as written after spending several iterations to bring the primal and dual solutions close together. A highly converged functional is needed since SPECTRUM.PY assumes that the would-be zeros are close to the local minima of (B18) which uses only the polynomial numerator. The second is to modify the script to use a nonpolynomial function minimizer, allowing us to multiply (B18) by the prefactor $\chi_{\ell}$ from (B9). The advantage is that when $\mathbf{y}$ acts on a full convolved block, the local minima are closer to the physical $\Delta_{k}$. As a result, punishing SDPB parameters are no longer required. For the data in Fig. 7, we have used the first approach and specified

$$
\begin{aligned}
& - \text { PRECISION }=660, \\
& - \text { DUALITYGAPTHERSHOLD }=1 \mathrm{e}-75
\end{aligned}
$$

as the nondefault parameters. The rest of the script obtains high precision OPE coefficients directly from the primal solution. ${ }^{20}$

To plot the points in Fig. 8, we have used an older method which minimizes the error in a set of crossing equations with known scaling dimensions. ${ }^{21}$ Once a set of $Z$ stable operators has been found, we may consider a truncated crossing equation of the form

\footnotetext{
${ }^{20}$ For an implementation with linear programming, see [45]. The semidefinite programming version first appeared in [80] which uses opposite conventions for what the primal and dual problems are.

${ }^{21} \mathrm{An}$ analogous approach to the severely truncated bootstrapfitting operator dimensions and OPE coefficients at the same time -was recently explored in [81].
}

$$
\sum_{k=1}^{Z} a_{k} \mathbf{F}_{k}=\mathbf{n}
$$

When studying a single correlator involving $\mathbb{Z}_{2}$-even operators, we make the identifications

$$
\begin{aligned}
a_{k} & =\lambda_{\phi \phi \mathcal{O}_{k}}^{2}, \\
\mathbf{F}_{k} & =\mathbf{F}_{-, \Delta_{k}, \ell_{k}}^{\phi \phi ; \phi \phi}, \\
\mathbf{n} & =-\mathbf{F}_{-,, 0,0}^{\phi \phi ; \phi \phi}
\end{aligned}
$$

The point is that $\phi$ may denote $\sigma, \epsilon$, or indeed any scalar whose self-OPE is well approximated by the $Z$ operators in our set. We regard (B19) as a set of $N>Z$ linear equations with $N$ given by (B6). A naive approach is to remove rows corresponding to high derivatives bringing the number of equations down to $Z$. This often leads to $a_{k}$ coefficients that are negative, even when we use dimensions that are accurate to four digits. To overcome this, we follow [29] and leave one extra equation so that $N=Z+1$. With this overdetermined system, we will not be able to find $a_{k}$ that satisfy (B19) exactly. Rather, we find the $a_{k}$ that minimize the distance between the left and right sides of (B19). In this fit, the constraint $a_{k} \geq 0$ may be specified by hand. If our norm for this is the 1-norm, it is convenient to introduce a vector of positive entries $\mathbf{t}$ such that

$$
\begin{gathered}
-\mathbf{t} \leq \mathbf{n}-\sum_{k=1}^{Z} a_{k} \mathbf{F}_{k} \leq \mathbf{t}, \\
\left\|\mathbf{n}-\sum_{k=1}^{Z} a_{k} \mathbf{F}_{k}\right\| \leq \sum_{k=1}^{Z+1} t_{k} .
\end{gathered}
$$

We may now concatenate $\mathbf{t}$ and $\mathbf{a}$ into a vector $\mathbf{y}$ and recognize that the norm (B21) is $\mathbf{b}^{\mathrm{T}} \mathbf{y}$ with the objective $\mathbf{b}^{\mathrm{T}}=[1, \ldots, 1,0, \ldots, 0]$. Under the identifications

$B=\left[\begin{array}{cccc}-I_{Z+1} & \mathbf{F}_{1} & \ldots & \mathbf{F}_{Z} \\ -I_{Z+1} & -\mathbf{F}_{1} & \ldots & -\mathbf{F}_{Z}\end{array}\right], \quad \mathbf{c}=\left[\begin{array}{c}\mathbf{n} \\ -\mathbf{n}\end{array}\right]$,

this becomes the linear program (LP):

$$
\begin{aligned}
& \text { minimize } \mathbf{b}^{\mathrm{T}} \mathbf{y} \text { over } \mathbf{y} \geq 0, \\
& \text { such that } B \mathbf{y} \leq \mathbf{c} .
\end{aligned}
$$

It is amusing to point out that a reader using SDPB may continue to use it for solving (B23) because every linear program is also a semidefinite program. To do this, the $(2 Z+2) \times(2 Z+1)$ and $(2 Z+2) \times 1$ matrices $B$ and $\mathbf{c}$ must be enlarged to $(4 Z+3) \times(2 Z+1)$ and $(4 Z+3) \times 1$ so that they encode component-wise positivity of $\mathbf{y}$ in addition to (B21). The next necessary step is replacing $\mathbf{b}$ 


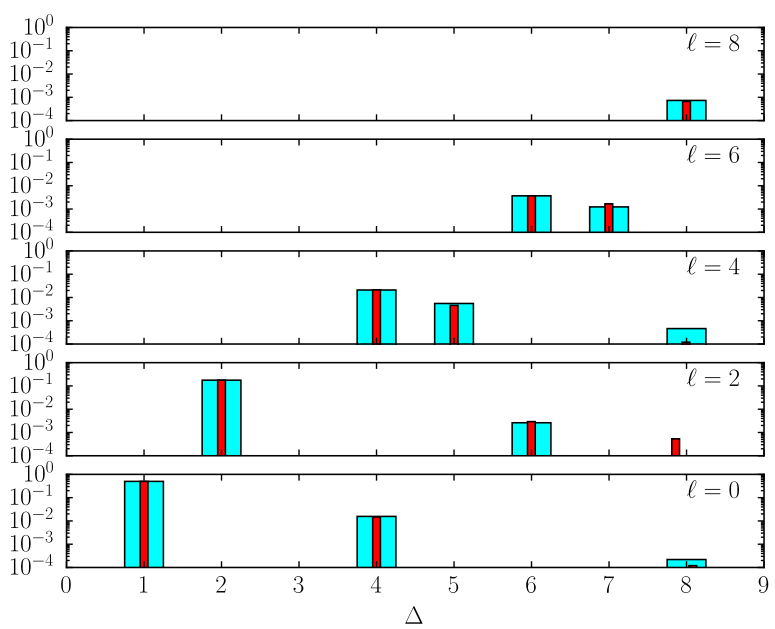

(a) $\left|\lambda_{\sigma \sigma \mathcal{O}}\right|$ comparison

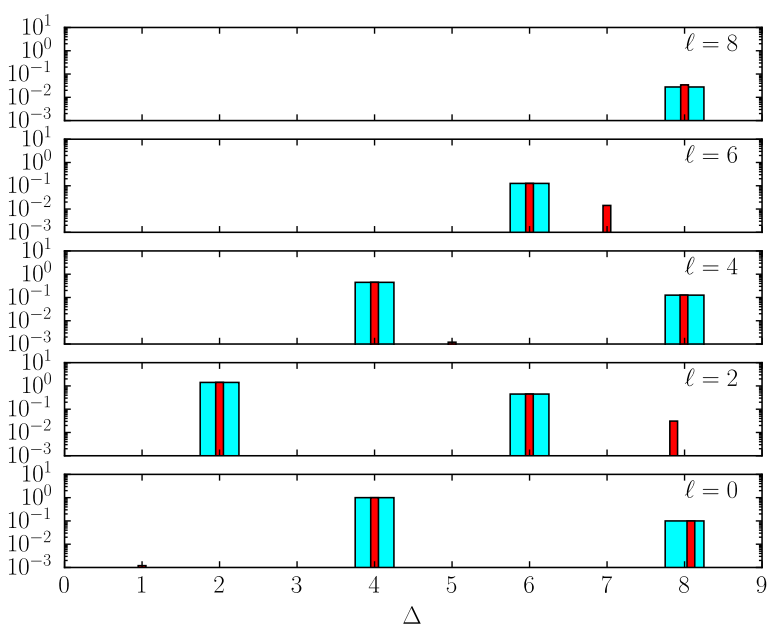

(b) $\left|\lambda_{\epsilon \epsilon \mathcal{O}}\right|$ comparison

FIG. 9. OPE coefficients in the 2D Ising model for operators with $\Delta \leq 8$. Wide blue bars show exact values and narrow red bars show estimates from the fit used in [29]. (a) Comparison for coefficients in the $\sigma \times \sigma$ OPE. (b) Comparison for coefficients in the $\varepsilon \times \varepsilon$ OPE.

with - b (and prepending an arbitrary number) as SDPB's objective is maximized instead of minimized. Finally, the orthogonal polynomials should all be 1 . With this choice, constraint matrices in (B17) simply pull off a diagonal component of $Y$ and $\mathbf{c}-B \mathbf{y}=\operatorname{Tr}\left(A_{*} Y\right)$ reads $\mathbf{c}-B \mathbf{y} \geq 0$. In any case, whether (B23) is solved as an LP or SDP, it is clear that the returned $\mathbf{y}$ will give us the OPE coefficients $\lambda_{\phi \phi \mathcal{O}_{k}}^{2}$.

To verify that this gives reliable values for $a_{k}$ in two dimensions, we have compared exact and approximate results in the Ising model where $\epsilon \times \epsilon$ operators are also in $\sigma \times \sigma$. Starting with $\Delta_{\phi}=\Delta_{\sigma}$, the $\lambda_{\sigma \sigma \mathcal{O}}^{2}$ coefficients are very close to the ones found in [29]. Changing the external dimension to $\Delta_{\phi}=\Delta_{\epsilon}$, the same algorithm produces accurate $\lambda_{\epsilon \epsilon \mathcal{O}}^{2}$ coefficients as well. These are shown in Fig. 9.

Turning to three dimensions, exact Ising CFT data are not available, but the tables in [47] have negligible error for our purposes. Despite all the progress in isolating this model, it appears that several spin- $\ell$ operators are still missing from these spectra: the ones that do not fall into $[\sigma \sigma]_{n},[\epsilon \epsilon]_{n}$, and $[\sigma \epsilon]_{n}$ twist families. As evidence of this, Fig. 10 shows that OPE coefficients fit with the dual method differ greatly from the ones returned by SPECTRUM.PY. To guess where the first missing operator appears, we can take the naive view that twist families exist all the way down to $\ell=0$. Constructing one out of an irrelevant operator $\Phi$, we have $\Delta \approx 2 \Delta_{\Phi}+2 n+\ell>2 d$.

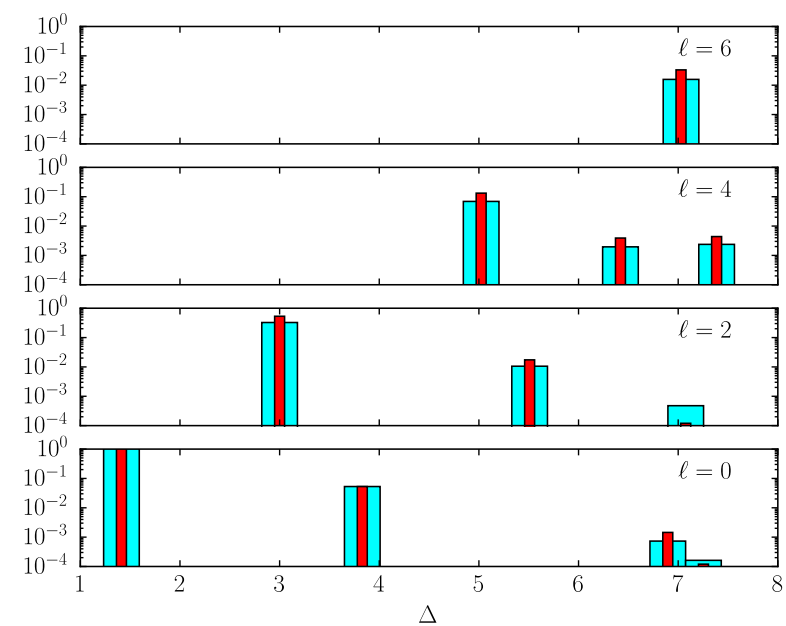

(a) $\left|\lambda_{\sigma \sigma \mathcal{O}}\right|$ comparison

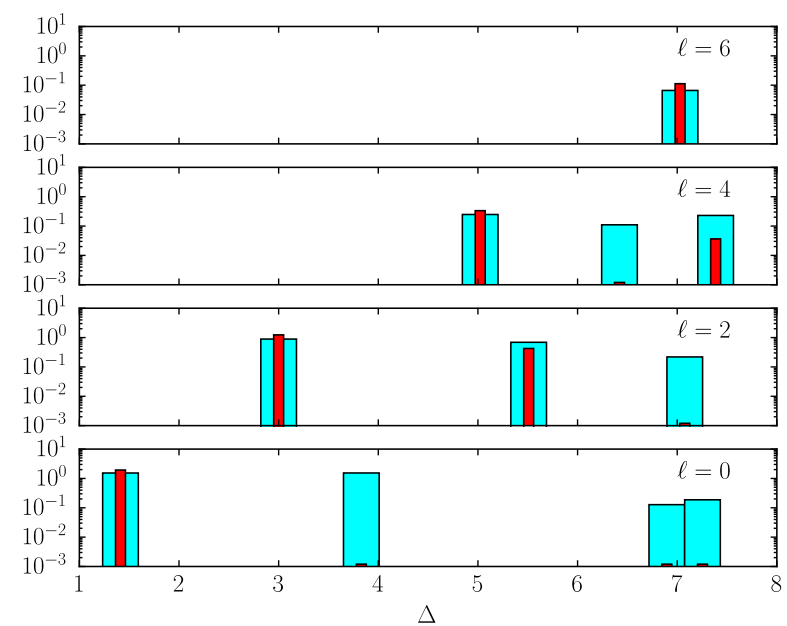

(b) $\left|\lambda_{\epsilon \epsilon \mathcal{O}}\right|$ comparison

FIG. 10. The analogue of Fig. 9 for the 3D Ising model. In the fit, we have used the $\mathbb{Z}_{2}$-even operator dimensions from [47]. This is already a longer list than anything that is likely to come from a one-correlator bootstrap. Due to a remaining bias in the spectrum, there is strong disagreement between the primal and dual methods. 
Four-point functions approximated by bootstrap data have already proven useful for conformal perturbation theory and measuring non-Gaussianity $[82,83]$. As it is significantly affected by the incompleteness of the spectrum above $\Delta_{*} \approx 2 d$, a fit to the crossing equations must be a less forgiving problem.

Although we have not done so in this work, it should also be possible to fit mixed OPE coefficients in a 2D theory. The last two rows of (B3) would lead to coupled quadratic equations which rapidly become impractical to solve as our system grows. Therefore, we will focus on the third row. If only the identity has been singled out, this is a homogenous equation and its parameters are ambiguous up to a rescaling. This is why it helps to find OPE coefficients in a prescribed order. As long as $\lambda_{\phi \phi \phi^{2}}$ has been found from the $\phi \times \phi$ fit described above, permutation symmetry of this coefficient may be used to make our equation inhomogeneous. In this case, it takes the form of (B19) but now with

$$
\begin{aligned}
a_{k} & =\lambda_{\phi \phi^{2} \mathcal{O}_{k}}^{2}, \\
\mathbf{F}_{k} & =\mathbf{F}_{-, \Delta_{k}, \ell_{k}}^{\phi \phi^{2} ; \phi^{2}}, \\
\mathbf{n} & =-\lambda_{\phi \phi^{2} \phi}^{2} \mathbf{F}_{-, \Delta_{\phi}, 0}^{\phi \phi^{2} ; \phi \phi^{2}} .
\end{aligned}
$$

Essentially, $\phi$ plays the same role that the identity operator played before.
[1] A. A. Belavin, A. M. Polyakov, and A. B. Zamolodchikov, Infinite conformal symmetry in two-dimensional quantum field theory, Nucl. Phys. B241, 333 (1984).

[2] D. Friedan, Z. Qiu, and S. Shenker, Conformal Invariance, Unitarity, and Critical Exponents in Two Dimensions, Phys. Rev. Lett. 52, 1575 (1984).

[3] D. Friedan, Z. Qiu, and S. Shenker, Details of the nonunitarity proof for highest weight representations of the Virasoro algebra, Commun. Math. Phys. 107, 535 (1986).

[4] P. Goddard, A. Kent, and D. Olive, Virasoro algebras and coset space models, Phys. Lett. 152B, 88 (1985).

[5] P. Goddard, A. Kent, and D. Olive, Unitary representations of the Virasor and super-Virasoro algebras, Commun. Math. Phys. 103, 105 (1986).

[6] R. Rattazzi, S. Rychkov, E. Tonni, and A. Vichi, Bounding scalar operator dimensions in 4D CFT, J. High Energy Phys. 12 (2008) 031.

[7] S. Rychkov and A. Vichi, Universal constraints on conformal operator dimensions, Phys. Rev. D 80, 045006 (2009).

[8] F. Caracciolo and S. Rychkov, Rigorous limits on the interaction strength in quantum field theory, Phys. Rev. D 81, 085037 (2010).

[9] R. Rattazzil, S. Rychkov, and A. Vichi, Central charge bounds in 4D conformal field theory, Phys. Rev. D 83, 046011 (2011).

[10] R. Rattazzil, S. Rychkov, and A. Vichi, Bounds in 4D conformal field theories with global symmetry, J. Phys. A 44, 035402 (2011).

[11] A. Vichi, Improved bounds for CFTs with global symmetries, J. High Energy Phys. 01 (2012) 162.

[12] D. Poland and D. Simmons-Duffin, Bounds on 4D conformal and superconformal field theories, J. High Energy Phys. 05 (2010) 017.

[13] D. Poland, D. Simmons-Duffin, and A. Vichi, Carving out the space of 4D CFTs, J. High Energy Phys. 05 (2012) 110.

[14] F. Kos, D. Poland, and D. Simmons-Duffin, Bootstrapping mixed correlators in the 3D Ising model, J. High Energy Phys. 11 (2014) 109.
[15] D. Simmons-Duffin, A semidefinite program solver for the conformal bootstrap, J. High Energy Phys. 06 (2015) 174.

[16] F. Kos, D. Poland, D. Simmons-Duffin, and A. Vichi, Precision islands in the Ising and $\mathrm{O}(\mathrm{N})$ models, J. High Energy Phys. 08 (2016) 036.

[17] P. Liendo, L. Rastelli, and B. C. van Rees, The bootstrap program for boundary $\mathrm{CFT}_{d}$, J. High Energy Phys. 07 (2013) 113.

[18] Al. B. Zamolodchikov, Three-point function in the minimal Liouville gravity, Theor. Math. Phys. 142, 183 (2005).

[19] A. A. Belavin and A. B. Zamolodchikov, Moduli integrals and ground ring in minimal Liouville gravity, J. Exp. Theor. Phys. Lett. 82, 7 (2005).

[20] S. Ribault, Conformal field theory on the plane, arXiv: 1406.4290 .

[21] M. Hogervorst and B. C. van Rees, Crossing symmetry in alpha space, J. High Energy Phys. 11 (2017) 193.

[22] M. Hogervorst, Crossing kernels for boundary and crosscap CFTs, arXiv:1703.08159.

[23] I. Esterlis, A. L. Fitzpatrick, and D. Ramirez, Closure of the operator product expansion in the non-unitary bootstrap, J. High Energy Phys. 11 (2016) 030.

[24] F. Gliozzi, More Constraining Conformal Bootstrap, Phys. Rev. Lett. 111, 161602 (2013).

[25] F. Gliozzi and A. Rago, Critical exponents of the 3d Ising and related models from the conformal bootstrap, J. High Energy Phys. 10 (2014) 042.

[26] F. Gliozzi, P. Liendo, M. Meineri, and A. Rago, Boundary and interface CFTs from the conformal bootstrap, J. High Energy Phys. 05 (2015) 036.

[27] D. Simmons-Duffin, TASI lectures on the conformal bootstrap, arXiv: 1602.07982.

[28] A. Vichi, A new method to explore conformal field theories in any dimension, Ph.D. thesis, École Polytechnique Fédérale de Lausanne, 2011.

[29] S. El-Showk and M. F. Paulos, Bootstrapping Conformal Field Theories with the Extremal Functional Method, Phys. Rev. Lett. 111, 241601 (2013). 
[30] R. Koekoek and R. F. Swarttouw, The Askey-scheme of hypergeometric orthogonal polynomials and its q-analogue, Rep. Faculty Technical Math. Informat. 94, 23(1996).

[31] R. Gopakumar, A. Kaviraj, K. Sen, and A. Sinha, A Mellin space approach to the conformal bootstrap, J. High Energy Phys. 05 (2017) 027.

[32] W. Chu, Analytical formulae for extended ${ }_{3} F_{2}$-series of Watson-Whipple-Dixon with two extra integer parameters, Math. Comput. 81, 467 (2012).

[33] D. Mazáč, Analytic bounds and emergence of $\mathrm{AdS}_{2}$ physics from the conformal bootstrap, J. High Energy Phys. 04 (2017) 146.

[34] S. Caron-Huot, Analyticity in spin in conformal theories, J. High Energy Phys. 09 (2017) 078.

[35] P. Desrosiers, L. Lapointe, and P. Mathieu, Superconformal field theory and Jack superpolynomials, J. High Energy Phys. 09 (2012) 037.

[36] A. A. Belavin, M. A. Bershtein, and G. M. Tarnopolsky, Bases in coset conformal field theory from AGT correspondence and Macdonald polynomials at the roots of unity, J. High Energy Phys. 03 (2013) 019.

[37] L. Alarie-Vezina, P. Desrosiers, and P. Mathieu, Ramond singular vectors and Jack superpolynomials, J. Phys. A 47, 035202 (2014).

[38] V. S. Dotsenko and V. A. Fateev, Conformal algebra and multipoint correlation functions in 2D statistical models, Nucl. Phys. B240, 312 (1984).

[39] V.S. Dotsenko and V. A. Fateev, Four point correlation functions and the operator algebra in the two-dimensional conformal invariant theories with central charge $c \leq 1$, Nucl. Phys. B251, 691 (1985).

[40] V. S. Dotsenko and V. A. Fateev, Operator algebra of twodimensional conformal theories with central charge $c \leq 1$, Phys. Lett. 154B, 291 (1985).

[41] J. A. Wilson, Three term contiguous relations and some new orthogonal polynomials, in Pade and Rational Approximations, edited by E. Saff and R. Varga (Academic Press, New York, 1977), pp. 227-232.

[42] P. Suchanek, Recursive methods of determination of 4-point blocks in $N=1$ superconformal field theories, Ph.D. thesis, Jagiellonian University, 2009.

[43] P. Furlan, A. C. Ganchev, and V. B. Petkova, Fusion matrices and $c<1$ (quasi) local conformal theories, Int. J. Mod. Phys. A 05, 2721 (1990).

[44] M. Pawelkiewicz, V. Schomerus, and P. Suchanek, The universal Racah-Wigner symbol for $U_{q}(\operatorname{OSp}(1 \mid 2))$, J. High Energy Phys. 04 (2014) 079.

[45] S. El-Showk, M. F. Paulos, D. Poland, S. Rychkov, D. Simmons-Duffin, and A. Vichi, Solving the 3D Ising model with the conformal bootstrap II: c-Minimization and precise critical exponents, J. Stat. Phys. 157, 869 (2014).

[46] S. El-Showk and M. F. Paulos, Extremal bootstrapping: Go with the flow, J. High Energy Phys. 03 (2018) 148.

[47] D. Simmons-Duffin, The lightcone bootstrap and the spectrum of the 3d Ising CFT, J. High Energy Phys. 03 (2017) 086.

[48] C.-M. Chang and Y.-H. Lin, Carving out the end of the world or (superconformal bootstrap in six dimensions), J. High Energy Phys. 08 (2017) 128.
[49] C.-M. Chang, M. Fluder, Y.-H. Lin, and Y. Wang, Spheres, charges, instantons and bootstrap: A five-dimensional odyssey, J. High Energy Phys. 03 (2018) 123.

[50] M. Cornagliotto, M. Lemos, and P. Liendo, Bootstrapping the $\left(A_{1}, A_{2}\right)$ Argyres-Douglas theory, J. High Energy Phys. 03 (2018) 033.

[51] S. Collier, P. Kravchuk, Y.-H. Lin, and X. Yin, Bootstrapping the spectral function: On the uniqueness of Liouville theory and the universality of BPZ, arXiv: 1702.00423.

[52] Y.-H. Lin, S.-H. Shao, D. Simmons-Duffin, Y. Wang, and $\mathrm{X}$. Yin, $N=4$ superconformal bootstrap of the K3 CFT, J. High Energy Phys. 05 (2017) 126.

[53] Y.-H. Lin, S.-H Shao, Y. Wang, and X. Yin, (2,2) superconformal bootstrap in two dimensions, J. High Energy Phys. 05 (2017) 112.

[54] M. Cornagliotto, M. Lemos, and V. Schomerus, Long multiplet bootstrap, J. High Energy Phys. 10 (2017) 119.

[55] M. F. Paulos, S. Rychkov, B. C. van Rees, and B. Zan, Conformal invariance in the long-range Ising model, Nucl. Phys. B902, 296 (2016).

[56] C. Behan, L. Rastelli, S. Rychkov, and B. Zan, A scaling theory for the long-range to short-range crossover and an infrared duality, J. Phys. A 50, 354002 (2017).

[57] C. Behan, L. Rastelli, S. Rychkov, and B. Zan, Long-Range Critical Exponents Near the Short-Range Crossover, Phys. Rev. Lett. 118, 241601 (2017).

[58] M. Hogervorst, S. Rychkov, and B. C. van Rees, Unitarity violation at the Wilson-Fisher fixed point in 4-epsilon dimensions, Phys. Rev. D 93, 125025 (2016).

[59] E. Dyer, A. L. Fitzpatrick, and Y. Xin, Constraints on flavored 2d CFT partition functions, J. High Energy Phys. 02 (2018) 148.

[60] S. Rychkov, Conformal bootstrap in three dimensions? arXiv:1111.2115.

[61] Z. Li and N. Su, 3D CFT archipelago from single correlator bootstrap, arXiv:1706.06960.

[62] V. Bashmakov, M. Bertolini, and H. Raj, On nonsupersymmetric conformal manifolds: Field theory and holography, J. High Energy Phys. 11 (2017) 167.

[63] M. Baggio, N. Bobev, S. M. Chester, E. Lauria, and S. S. Pufu, Decoding a three-dimensional conformal manifold, J. High Energy Phys. 02 (2018) 062.

[64] C. Behan, Conformal manifolds: ODEs from OPEs, J. High Energy Phys. 03 (2018) 127.

[65] S. Hollands, Action principle for OPE, Nucl. Phys. B926, 614 (2018).

[66] A. L. Fitzpatrick, J. Kaplan, D. Poland, and D. SimmonsDuffin, The analytic bootstrap and AdS superhorizon locality, J. High Energy Phys. 12 (2013) 004.

[67] Z. Komargodski and A. Zhiboedov, Convexity and liberation at large spin, J. High Energy Phys. 11 (2013) 140.

[68] D. Friedan, Z. Qiu, and S. Shenker, Superconformal invariance in two dimensions and the tricritical Ising model, Phys. Lett. 151B, 37 (1985).

[69] Z. Qiu, Supersymmetry, two-dimensional critical phenomena and the tricritical Ising model, Nucl. Phys. B270, 205 (1986).

[70] J. Rong and N. Su, Scalar CFTs and their large N limits, arXiv:1712.00985. 
[71] A. C. Echeverri, B. von Harling, and M. Serone, The effective bootstrap, J. High Energy Phys. 09 (2016) 097.

[72] R. Wong, Five lectures on asymptotic theory, in Differential Equations and Asymptotic Theory in Mathematical Physics, edited by Z. Hua and R. Wong (World Scientific, Singapore, 20040, pp. 189-263.

[73] D. Pappadopulo, S. Rychkov, J. Espin, and R. Rattazzi, OPE convergence in conformal field theory, Phys. Rev. D 86, 105043 (2012).

[74] S. Rychkov and P. Yvernay, Remarks on the convergence properties of the conformal block expansion, Phys. Lett. B 753, 682 (2016).

[75] E.X.W. Xia and X. M. Yao, The signs of three-term recurrence sequences, Discrete Appl. Math. 159, 2290 (2011).

[76] T. Hartman, S. Jain, and S. Kundu, Causality constraints in conformal field theory, J. High Energy Phys. 05(2016) 099.
[77] M. Hogervorst and S. Rychkov, Radial coordinates for conformal blocks, Phys. Rev. D 87, 106004 (2013).

[78] M. Hogervorst, H. Osborn, and S. Rychkov, Diagonal limit for conformal blocks in $d$ dimensions, J. High Energy Phys. 08 (2013) 014.

[79] C. Behan, PyCFTBoot: A flexible interface for the conformal bootstrap, Commun. Comput. Phys. 22, 1 (2017).

[80] C. Beem, M. Lemos, P. Liendo, L. Rastelli, and B. C. van Rees, The $\mathcal{N}=2$ superconformal bootstrap, J. High Energy Phys. 03 (2016) 183.

[81] W. Li, New method for conformal bootstrap with OPE truncations, arXiv:1711.09075.

[82] Z. Komargodski and D. Simmons-Duffin, The random-bond Ising model in 2.01 and 3 dimensions, J. Phys. A 50, 154001 (2017).

[83] S. Rychkov, D. Simmons-Duffin, and B. Zan, Nongaussianity of the critical 3d Ising model, SciPost Phys. 2, 001 (2017). 